\title{
Optimal Time-Consistent Investment Strategy for a Random Household Expenditure with Default Risk under Relative Performance
}

\author{
Wenjin Guan, ${ }^{1}$ Wei Yuan, ${ }^{1}$ and Sheng $\mathrm{Li} \mathbb{1}^{2}$ \\ ${ }^{1}$ Sichuan Administration Institute, Chengdu 610072, China \\ ${ }^{2}$ School of Statistics, Chengdu University of Information Technology, Chengdu 610103, China
}

Correspondence should be addressed to Sheng Li; lisheng@cuit.edu.cn

Received 29 April 2021; Revised 13 August 2021; Accepted 18 August 2021; Published 8 October 2021

Academic Editor: Baogui Xin

Copyright (C) 2021 Wenjin Guan et al. This is an open access article distributed under the Creative Commons Attribution License, which permits unrestricted use, distribution, and reproduction in any medium, provided the original work is properly cited.

Considering the mind of rivalry between families, each family focuses not only on its own wealth but also on other families, especially neighbors. In this paper, we investigate the non-zero-sum mean-variance game between two families with a random household expenditure under the default risk and relative performance. Applying the stochastic control theory within the framework of the game theory, the extended Hamilton-Jacobi-Bellman equation equations are derived. By solving this equation, we obtain the Nash equilibrium strategies of the two families and the corresponding equilibrium value functions. We also provide a numerical example to analyze the effects of relevant parameters on Nash equilibrium strategy and on the utility loss due to the mind of rivalry.

\section{Introduction}

With the strengthening of household residents' financial awareness and the increasing abundance of financial products, families are increasingly focusing on the issue of optimal asset portfolio selection. Optimal investment portfolios have been widely studied in recent years. Hipp and Plum [1], David Promislow and Young [2], Milevsky et al. [3], Chen et al. [4], and Bayraktar and Zhang [5] consider the optimal investment for minimizing the ruin probability. Wang [6], Bo and Capponi [7], Delong [8], Yuan and Lai [9], and $\mathrm{Li}$ et al. [10] obtain the optimal investment strategy under maximizing the expected utility of terminal wealth.

And then the mean-variance criteria are another objective of great interest to insurers. In contrast to maximizing expected utility and minimizing ruin probability, meanvariance criteria allow insurers to minimize risk at an acceptable return. This approach takes not only the risk but also the returns into account. Due to its rationality and practicality, mean-variance has become a popular decision criterion in financial theory. The study of the mean-variance can be traced back to Markowitz [11]. Since then, there has been a certain amount of research on the optimization problem and its application in finance and insurance. For example, $\mathrm{Li}$ and $\mathrm{Ng}$ [12] introduce an embedding technique to transform the mean-variance problem into a stochastic linear quadratic control problem in a discrete-time model, which was extended to the corresponding continuous-time model by Zhou and Li [13]. The application of the meanvariance criterion to optimal reinsurance or/and investment can be found in Bäuerle [14], Bai and Zhang [15], and Bi and Guo [16]; however, they are all in the optimal strategy based on initial information, which is time-inconsistent. It is well known that the dynamic mean-variance criterion lacks iterated-expectation property, which leads to time-inconsistent in the sense that the Bellman optimality principle does not hold anymore. To this end, Björk et al. [17] solve the time-inconsistent problem by reconstructing stochastic control theory in the framework of game theory and obtain a time-consistent equilibrium strategy which is not only 
optimal at the current time but also optimal in the future. In recent years, there has been an increased interest in finding a time-consistent equilibrium strategy for the mean-variance portfolio problem, such as Zeng and Li [18], Li et al. [19], and Zhang and Liang [20].

Further, high-yield corporate bonds are becoming increasingly attractive to investors in today's financial markets. As a result, the optimal portfolio problem with defaulted securities has become an important area of research. Bielecki and Jang [21] consider the portfolio problem of investing wealth in credit risk assets, bank accounts, and stocks to maximize exponential utility maximization. Li et al. [22] investigate the $\mathrm{DC}$ pension management problem with a default bond under the CEV model and obtain an optimal time-consistent investment strategy. Zhao et al. [23] and Zhang and Chen [24] derive the optimal time-consistent investment and reinsurance strategies under default risk.

Similar to Yuan and Lai [9] and Li et al. [10], we study households with stochastic expenditure optimal investment strategies. Unlike them, we consider that households can invest in corporate bonds and study the optimal timeconsistent strategy under the mean-variance. Further, relative performance concerns are prevalent, such as pension managers, insurers, and other firms, who are concerned not only with their absolute wealth but also with the gap between their wealth and that of their competitors. And considering that there is a mind of rivalry among families, and they not only focus on their own wealth but also on the wealth of other families, especially their neighbors; thus, we construct a non-zero-sum mean-variance game between two families under relative performance (see Espinosa and Touzi [25]). Applying the stochastic control theory in the game theory framework, we obtain the Nash equilibrium strategies of the two households and the corresponding equilibrium value functions, and in addition, we analyze the utility loss caused by the mind of rivalry.

The remainder of this paper is organized as follows. Section 2 formulates the mean-variance game between two families for a random household expenditure with default risk. In Section 3, by solving the extended HJB equation, we derive the time-consistent Nash equilibrium strategy and the corresponding equilibrium value functions. In Section 4, we provide a numerical example to simulate the influence of relevant parameters on the equilibrium strategy and give an economic explanation. Section 5 concludes this paper.

\section{Problem Formulation}

In this paper, we consider a complete probability space $(\Omega, \mathscr{F}, \mathbb{P})$ equipped with a right continuous and $\mathbb{P}$-complete filtration $\left\{\mathscr{F}_{t}\right\}_{t \in[0, T]}$, where $\left\{\mathscr{F}_{t}\right\}_{t \in[0, T]}$ represents the information of the market available up to time $t$. All stochastic processes introduced below are defined in the filtered probability space $\left(\Omega, \mathscr{F},\left\{\mathscr{F}_{t}\right\}_{t \in[0, T]}, \mathbb{P}\right)$.

Suppose that the financial market consists of three tradable assets: a risk-free asset, a risky asset, and a defaultable bond. The price of a risk-free asset satisfies the following process:

$$
\mathrm{d} R(t)=r R(t) \mathrm{d} t, \quad R(0)=R_{0},
$$

where $r(>0)$ is the risk-free interest rate. The price $S(t)$ of the stock is given by

$$
\mathrm{d} S(t)=S(t-)\left[\mu \mathrm{d} t+\sigma \mathrm{d} W_{1}(t)+\mathrm{d} \sum_{i=1}^{N(t)} Y_{i}\right], \quad S(0)=S_{0}
$$

where $\mu(>0)$ and $\sigma(>0)$ are the expected return rate and the instantaneous volatility, respectively. $\{N(t)\}_{t \in[0, T]}$, denoting the number of the price jumps that occur in the risky asset during time interval $[0, t]$, is a homogeneous Poisson process with an intensity $\lambda ; Y_{l}$ is the $l$ th jump amplitude of the risky asset price; $\left\{Y_{l}, l=1,2, \ldots\right\}$ are i.i.d random variables with distribution function $F(y)$, finite first-order moment $E\left[Y_{l}\right]=\mu_{Y}$, and second-order moment $E\left[Y_{l}^{2}\right]=\sigma_{Y}^{2}$. Besides, we suppose that $\mathbb{P}\left\{Y_{l} \geq-1\right.$ for all $l \geq 1\}=1$ to ensure that the risky asset price remains positive. And we assume that $\mu+\lambda \mu_{Y}>r$.

Different from the price dynamics of the risk-free asset and risky asset which are defined under the real-world probability measure $\mathbb{P}$ directly, the price process of the defaultable bond is firstly given by the martingale probability measure $\mathbb{Q}$ and then turned into the price dynamics under the probability measure $\mathbb{P}$. Following Bielecki and Jang [21], the definition of the default process is given by $Z(t)=1_{\{\tau \leq t\}}$, where $\tau$ is a nonnegative random variable representing the default time of the company issue the bond, 1 is an indicator which takes value one if the default occurs, and zero otherwise. That is, the default process $Z(t)$ is nondecreasing right continuous and has a jump at the random time $\tau$. Furthermore, according to Driessen [26] and Duffie and Singleton [27], $Z(t)$ is supposed as the default process with a constant intensity $k^{\mathbb{P}}$ under the probability measure $\mathbb{P}$. Then, the martingale default process $K^{\mathbb{P}}(t)$ under probability measure $\mathbb{P}$ is given by $K^{\mathbb{P}}(t):=Z(t)-\int_{0}^{t}(1-Z(u-)) k^{\mathbb{P}} \mathrm{d} u$, where its differential form is $\mathrm{d} K^{\mathbb{P}}(t)=\mathrm{d} Z(t)-k^{\mathbb{P}}(1-$ $Z(t-)) \mathrm{d} t$.

Then, the price process of the defaultable bond under the martingale probability measure $\mathbb{Q}$ is considered. Similar to Bielecki and Jang [21], the defaultable bond is assumed as a zero-coupon bond with the one-unit face value and maturity $\bar{T}$. Further, we assume that in the event that a default occurs, the insurer recovers a fraction of the market value of the defaultable bond just prior to default, and then, the postdefault value of the defaultable bond is zero. The loss rate is denoted by $\zeta$; then, $1-\zeta$ is the default recovery rate. In addition, we denote by $k^{\mathbb{Q}}$ the constant intensity of the default process under the martingale probability measure $\mathbb{Q}$, then $\vartheta=k^{\mathbb{Q}} \zeta$ is the risk-neutral credit spread, and then, we derive the following price process of the defaultable bond under the probability measure $\mathbb{Q}$ :

$$
p(t, \bar{T})=1_{\tau>t} e^{-(r+9)(\bar{T}-t)}+1_{\tau \leq t}(1-\zeta) e^{-(t+\vartheta)(\bar{T}-t)} e^{r(t-\tau)},
$$

where $p(t, \bar{T})$ is a fictitious bond rather than a bond actually traded, and $T<\bar{T}$. That is, as Bielecki and Jang [21] and 
Driessen [26] mentioned, the fictitious bond allows us to take into consideration the jump risk premium in the expected return of the defaultable bond. Applying Itô formula to the above expression, we derive

$$
\mathrm{d} p(t, \bar{T})=r p(t, \bar{T}) \mathrm{d} t-\zeta e^{-(r+9)(\bar{T}-t)} \mathrm{d} K^{\mathbb{Q}}(t),
$$

where $K^{\mathbb{Q}}(t)$ is a martingale default process under the martingale probability measure $\mathbb{Q}$.
Next, we need to transform the price process of defaultable bonds under the martingale probability measure $\mathbb{Q}$ into the real-world probability measure $\mathbb{P}$. Based on the above notations, by Girsanov theorem (see Kusuoka [28]), there exists a predictable process $\Delta$ such that the evolutions of the price process for the defaultable bond under realworld probability measure $\mathbb{P}$ as follows:

$$
\mathrm{d} p(t, \bar{T})=p(t-, \bar{T})\left[r \mathrm{~d} t+(1-Z(t)) \vartheta(1-\Delta) \mathrm{d} t-(1-Z(t-)) \zeta \mathrm{d} K^{\mathbb{P}}(t)\right],
$$

where $\quad \Delta=\left(k^{\mathbb{Q}} / k^{\mathbb{P}}\right) \quad$ and $\quad K^{\mathbb{P}}(t)=Z(t)-k^{\mathbb{Q}} \int_{0}^{t}(1-Z$ $(u-)) \Delta \mathrm{d} u$ is a $\mathscr{F}$-martingale under probability measure $\mathbb{P}$. Similar to Duffie and Singleton [27], the probability of default under the real-world probability measure $\mathbb{P}$ is lower than that under the martingale probability measure $\mathbb{Q}$, that is, $(1 / \Delta)>1$. According to Yu [29], equation (5) consists of two components: the first component is the return of a default-free bond. The second is the difference between the risk-neutral credit spread and the real-world credit spread provided that the default has not occurred by time $t$.

In addition, we assume that household expenditures include household consumption expenditures, transaction costs, and other expenditures. Thus, the family expenditure has stochastic property. We denote the family expenditure at the time $t$ by $w(t)$ which is characterized by an arithmetic Brownian motion:

$$
\mathrm{d} w(t)=\alpha \mathrm{d} t+\beta \mathrm{d} W_{2}(t), \quad w(0)=w_{0}>0,
$$

where $\alpha(>0)$ represents the average expenditure. $\beta \mathrm{d} W_{2}(t)$ denotes expenditure subject to random factors, in which $\beta(>0)$ and $W_{2}(t)$ are positive constants and standard Brownian motion, respectively. And the correlation coefficient of $W_{1}(t)$ and $W_{2}(t)$ is denoted by $\rho$, i.e., $E\left[W_{1}(t) W_{2}(t)\right]=\rho t$. To simplify the calculation later, we assume that the expenditures are the same between the two households. In practice, the expenditures of the two households may be different, but it does not bring essential changes to the solution of the optimization problem but only increases the variables and parameters in the optimal solution.

Let $X_{i}(t)$ denote the wealth at the time $t \in[0, T]$ for family $i$, and $p_{i}(t)$ and $q_{i}(t)$ denote the amount at time $t$ for family $i$ invested in the risky asset and defaultable bond, respectively; then, the amount for family $i$ invested in riskfree assets is $X_{i}(t)-p_{i}(t)-q_{i}(t)$. Define $\pi_{i}(t)=\left(p_{i}(t)\right.$, $\left.q_{i}(t)\right)$ as the strategy of family $i$ at time $t$; thus, the dynamics of the wealth of family $i$ under strategy $\pi_{i}$ satisfies the following stochastic differential equation (SDE):

$$
\begin{aligned}
\mathrm{d} X_{i}^{\pi_{i}}(t)= & {\left[r X_{i}^{\pi_{i}}(t)+p_{i}(t)(\mu-r)+q_{i}(t)(1-Z(t)) \vartheta(1-\Delta)\right] \mathrm{d} t+p_{i}(t) \sigma \mathrm{d} W_{1}(t) } \\
& +\int_{-1}^{+\infty} p_{i}(t) y N(\mathrm{~d} t, \mathrm{~d} y)-q_{i}(t)(1-Z(t-)) \zeta \mathrm{d} K^{\mathbb{P}}-w(t) \mathrm{d} t, \quad X(0)=X_{0} .
\end{aligned}
$$

Definition 1 (admissible strategy). For any fixed $t \in[0, T]$, a strategy $\pi_{i}=\left\{\left(p_{i}(t), q_{i}(t)\right)\right\}_{t \in[0, T]}$ is said to be admissible if

(i) $\pi_{i}$ is $\mathscr{F}$-progressively measurable

(ii) $E\left[\int_{0}^{T}\left(\left(p_{i}(u)\right)^{2}+\left(q_{i}(u)\right)^{2}\right) \mathrm{d} u\right]<\infty$

(iii) The SDE (7) has a unique strong solution

For any initial state $\left(t, x_{i}, w, z\right) \in[0, T] \times \mathbb{R} \times \mathbb{R} \times\{0,1\}$, the corresponding set of all admissible strategies for family $i$ is denoted by $\Pi_{i}$. When default has occurred, i.e., $\tau \leq t$, we assume that $p(t-, \bar{T})=0$ and fix $p_{2}(t)=0$ afterward. And we let $z$ denote the initial default state, where $z=1$ and $z=0$ correspond to the postdefault cases $\tau>t$ and the predefault case $\tau \leq t$, respectively.
Due to the mind of rivalry, each family is concerned not only with their own terminal wealth but also with the differences between them and other families, especially neighbors. Hence, we formulate this optimization problem as a non-zero-sum stochastic differential game among two households. Following Espinosa and Touzi [25], we define the relative performance $\widehat{X}_{i}^{\pi_{i}}(t)$ for family $i$ as follows:

$$
\widehat{X}_{i}^{\pi_{i}, \pi_{j}}(t)=\left(1-\kappa_{i}\right) X_{i}^{\pi_{i}}(t)+\kappa_{i}\left(X_{i}^{\pi_{i}}(t)-X_{j}^{\pi_{j}}(t)\right), \quad i \neq j \in\{1,2\},
$$

where $\kappa_{i} \in[0,1]$ captures the intensity of the family $i$ 's relative concern and measures his sensitivity to the performance of his competitors. Together with equations (7) and (8), we derive 


$$
\begin{aligned}
\mathrm{d} \widehat{X}_{i}^{\pi_{i}, \pi_{j}}(t)= & {\left[r \widehat{X}_{i}^{\pi_{i}, \pi_{j}}(t)+(\mu-r)\left(p_{i}(t)-\kappa_{i} p_{j}(t)\right)+(1-Z(t)) \vartheta(1-\Delta)\left(q_{i}(t)-\kappa_{i} q_{j}(t)\right)-(1)-\kappa_{i} w(t)\right] \mathrm{d} t } \\
& +\left(p_{i}(t)-\kappa_{i} p_{j}(t)\right) \sigma \mathrm{d} W_{1}(t)+\int_{-1}^{+\infty}\left(p_{i}(t)-\kappa_{i} p_{j}(t)\right) N(\mathrm{~d} t, \mathrm{~d} y) \\
& -\left(q_{i}(t)-\kappa_{i} q_{j}(t)\right)(1-Z(t)) \zeta \mathrm{d} K^{\mathbb{P}}(t) .
\end{aligned}
$$

We assume that each family has mean-variance criteria and define the following value function for family $i$ :

$$
J_{i}^{\pi_{i}^{*}, \pi_{j}^{*}}\left(t, \widehat{x}_{i}, w, z\right)=\sup _{\pi_{i} \in \Pi_{i}}\left\{E_{t, \widehat{x}_{i}, w, z}\left[\widehat{X}_{i}^{\pi_{i}, \pi_{j}^{*}}(T)\right]-\frac{\gamma_{i}}{2} \operatorname{Var}_{t, \widehat{x}_{i}, w, z}\left[\widehat{X}_{i}^{\pi_{i}, \pi_{j}^{*}}(T)\right]\right\},
$$

where $E_{t, \widehat{x}_{i}, w, z}[\cdot]$ and $E_{t, \widehat{x}_{i}, w, z}[\cdot]$ are the conditional expectation and variance given $\hat{X}_{i}^{\pi_{i}, \pi_{j}}(t)=\hat{x}_{i}, w(t)=w$, and $Z(t)=z$ for $\left(t, \hat{x}_{i}, w, z\right) \in[0, T] \times \mathbb{R} \times \mathbb{R} \times\{0,1\}$, and $\gamma_{i}>0$ is the risk-aversion coefficient of family $i$.

Problem 1. In the classical non-zero-sum stochastic differential game, we find a Nash equilibrium $\left(\pi_{i}, \pi_{j}\right) \in \Pi_{i} \times$ $\Pi_{j}$ such that

$$
J_{i}^{\left(\pi_{i}^{*}, \pi_{j}^{*}\right)}\left(t, \widehat{x}_{i}, w, z\right) \geq J_{i}^{\left(\pi_{i}, \pi_{j}^{*}\right)}\left(t, \widehat{x}_{i}, w, z\right), \quad i \neq j \in\{1,2\} .
$$

Because there exists a nonlinear function of the expectation of terminal wealth in the objective functional (10), the optimization problem is time-inconsistent. In fact, time consistency cannot be ignored for a rational decision-maker who aims to seek an equilibrium strategy that is optimal at a time and still can be optimal as time goes forward into the future. Hence, we shall define the following time-consistent equilibrium strategy according to Björk et al. [17].

Definition 2. Consider an admissible strategy $\pi_{i}^{*}$ for family $i$, which can be informally viewed as a candidate equilibrium strategy. And choose arbitrarily a fixed $\widehat{\pi}_{i} \in \Pi_{i}$, a real number $\varepsilon(>0)$, and a given initial point $\left(t, \hat{x}_{i}, w, z\right) \in[0, T] \times \mathbb{R} \times \mathbb{R} \times\{0,1\} ;$ the strategy $\pi_{i}^{\varepsilon}$ for family $i$ is defined as follows:

$$
\pi_{i}^{\varepsilon}= \begin{cases}\hat{\pi}_{i}\left(u, \hat{x}_{i}, w, z\right), & \left(u, \hat{x}_{i}, w, z\right) \in[t, t+\varepsilon) \times \mathbb{R} \times \mathbb{R} \times\{0,1\}, \\ \pi_{i}^{*}\left(u, \widehat{x}_{i}, w, z\right), & \left(u, \widehat{x}_{i}, w, z\right) \in[t+\varepsilon, T] \times \mathbb{R} \times \mathbb{R} \times\{0,1\} .\end{cases}
$$

$$
\text { If }
$$

$$
\liminf _{\varepsilon \downarrow 0} \frac{J_{i}^{\left(\pi_{i}^{*}, \pi_{j}^{*}\right)}\left(t, \widehat{x}_{i}, w, z\right)-J_{i}^{\left(\pi_{i}^{\varepsilon}, \pi_{j}^{*}\right)}\left(t, \widehat{x}_{i}, w, z\right)}{\varepsilon} \geq 0,
$$

we call that $\pi_{i}^{*}$ is an equilibrium strategy of family $i$ and the equilibrium value function of family $i$ is given by $J_{i}^{\left(\pi_{i}^{*}, \pi_{j}^{*}\right)}\left(t, \widehat{x}_{i}, w, z\right)$.

\section{Nash Equilibrium Strategies}

In this section, we derive the Nash equilibrium strategy between two families. According to Definition 2, the equilibrium strategy above is time-consistent. We aim to seek an equilibrium strategy $\left(\pi_{1}^{*}, \pi_{2}^{*}\right)$ and the corresponding to equilibrium value functions. To give the extended HJB system and verification theorem conveniently, we define a variational operator. Let $C^{1,2,2}([0, T] \times \mathbb{R} \times \mathbb{R} \times\{0,1\})$ denote a space of any function $\varphi\left(t, \widehat{x}_{i}, w, z\right)$ which $\varphi\left(t, \widehat{x}_{i}, w, z\right)$ itself and its derivatives $(\partial \varphi / \partial t),\left(\partial \varphi / \partial \widehat{x}_{i}\right),\left(\partial^{2} \varphi / \partial \widehat{x}_{i}^{2}\right)$, $(\partial \varphi / \partial w),\left(\partial^{2} \varphi / \partial w^{2}\right)$, and $\left(\partial^{2} \varphi / \partial \hat{x}_{i} \partial w\right)$ are continuous on $[0, T] \times \mathbb{R} \times \mathbb{R} \times\{0,1\}$. For any function $\varphi\left(t, \widehat{x}_{i}, w, z\right) \in C^{1,2,2}([0, T] \times \mathbb{R} \times \mathbb{R} \times\{0,1\})$, the variational operator is defined as follows:

$$
\begin{aligned}
\mathscr{L}^{\left(\pi_{i}, \pi_{j}\right)} \varphi\left(t, \hat{x}_{i}, w, z\right)= & \frac{\partial \varphi}{\partial t}+\left[r \widehat{x}_{i}+(\mu-r)\left(p_{i}(t)-\kappa_{i} p_{j}(t)\right)-\left(1-\kappa_{i}\right) w\right. \\
& \left.+(1-z) \vartheta\left(q_{i}(t)-\kappa_{i} q_{j}(t)\right)\right] \frac{\partial \varphi}{\partial \widehat{x}_{i}}+\alpha \frac{\partial \varphi}{\partial w}+\frac{1}{2}\left(p_{i}(t)-\kappa_{i} p_{j}(t)\right)^{2} \sigma^{2} \frac{\partial^{2} \varphi}{\partial \widehat{x}_{i}^{2}}+\frac{1}{2} \beta^{2} \frac{\partial^{2} \varphi}{\partial w^{2}} \\
& +\rho \beta \sigma\left(p_{i}(t)-\kappa_{i} p_{j}(t)\right) \frac{\partial^{2} \varphi}{\partial \widehat{x}_{i} \partial w}+\lambda E\left[\varphi\left(t, \widehat{x}_{i}+\left(p_{i}(t)-\kappa_{i} p_{j}(t)\right) y, w, z\right)-\varphi\left(t, \widehat{x}_{i}, w, z\right)\right] \\
& +k^{\mathbb{P}}(1-z)\left[\varphi\left(t, \widehat{x}_{i}-\left(q_{i}(t)-\kappa_{i} q_{j}(t)\right) \zeta, w, 1\right)-\varphi\left(t, \widehat{x}_{i}, w, 0\right)\right] .
\end{aligned}
$$


Before finding the equilibrium strategy, the following theorem gives the verifications for the extended HJB equation corresponding to Problem 1.
Theorem 1. (verification theorem). For Problem 1, we assume that there exist two real-valued functions $V_{i}\left(t, \widehat{x}_{i}, w, z\right)$, $g_{i}\left(t, \hat{x}_{i}, w, z\right) \in C^{1,2,2}([0, T] \times \mathbb{R} \times \mathbb{R} \times\{0,1\})$ satisfying the following extended $H J B$ equations:

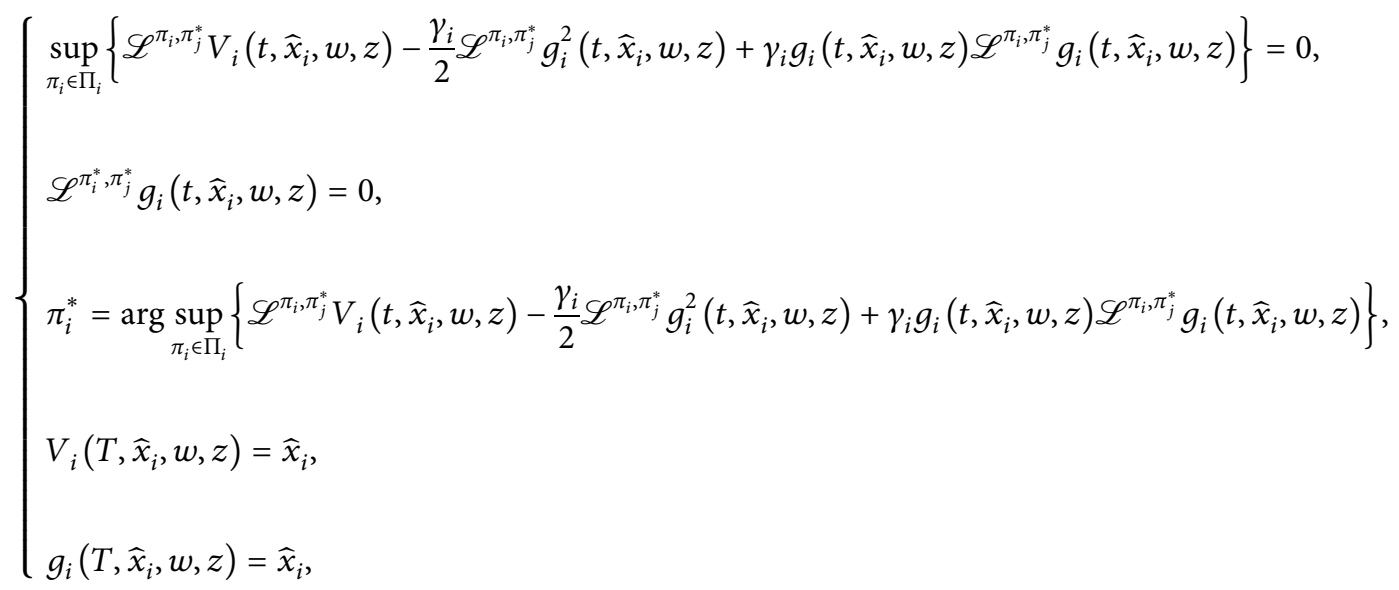

then $E_{t, \widehat{x}_{i}, w, z}\left[\widehat{X}_{i}^{\pi_{i}^{*}, \pi_{j}^{*}}(T)\right]=g_{i}\left(t, \hat{x}_{i}, w, z\right), \quad J_{i}^{\left(\pi_{i}^{*}, \pi_{j}^{*}\right)}\left(t, \hat{x}_{i}, w, z\right)$ $=V_{i}\left(t, \hat{x}_{i}, w, z\right)$, and $\pi_{i}^{*}$ is an equilibrium strategy of family $i$.
Theorem 2. For Problem 1, the optimal time-consistent Nash equilibrium strategies for family $i(\neq j \in\{1,2\})$ are given by

Proof. The proof of the verification theorem can be adapted from Theorem 5.2 in [17], and thus the proof is placed in Appendix A.

$$
\begin{aligned}
p_{i}^{*}(t)= & \frac{e^{-r(T-t)}}{1-\kappa_{i} \kappa_{j}}\left[\frac{r\left(\mu-r+\lambda \mu_{Y}\right)+\left(1-\kappa_{i}\right) \gamma_{i} \rho \beta \sigma\left(e^{r(T-t)}-1\right)}{\gamma_{i} r\left(\sigma^{2}+\lambda \sigma_{Y}^{2}\right)}\right. \\
& \left.+\kappa_{i} \frac{r\left(\mu-r+\lambda \mu_{Y}\right)+\left(1-\kappa_{j}\right) \gamma_{j} \rho \beta \sigma\left(e^{r(T-t)}-1\right)}{\gamma_{j} r\left(\sigma^{2}+\lambda \sigma_{Y}^{2}\right)}\right], \\
q_{i}^{*}(t)= & \frac{e^{-r(T-t)}}{1-\kappa_{i} \kappa_{j}}\left[\frac{\vartheta}{\gamma_{i} \zeta^{2} k^{\mathbb{P}}}-\frac{1}{\gamma_{i} \zeta}+\frac{c_{i}(t)-\bar{c}_{i}(t)}{\zeta}+\kappa_{i}\left(\frac{\vartheta}{\gamma_{j} \zeta^{2} k^{\mathbb{P}}}-\frac{1}{\gamma_{j} \zeta}+\frac{c_{j}(t)-\bar{c}_{j}(t)}{\zeta}\right)\right] 1_{\{\tau>t\}},
\end{aligned}
$$

and the equilibrium value function of family $i$ is

$$
V_{i}\left(t, \hat{x}_{i}, w, z\right)= \begin{cases}e^{r(T-t)} \widehat{x}_{i}+B_{i}(t) w+C_{i}(t), & z=1, \\ e^{r(T-t)} \widehat{x}_{i}+\bar{B}_{i}(t) w+\bar{C}_{i}(t), & z=0 .\end{cases}
$$

Moreover, the expectation and variance of the terminal value corresponding to the equilibrium strategy for family $i$ are as follows:

$$
\begin{aligned}
& E_{t, \widehat{x}_{i}, w, z}= \begin{cases}e^{r(T-t)} \widehat{x}_{i}+b_{i}(t) w+c_{i}(t), & z=1, \\
e^{r(T-t)} \widehat{x}_{i}+\bar{b}_{i}(t) w+\bar{c}_{i}(t), & z=0,\end{cases} \\
& \operatorname{Var}_{t, \hat{x}_{i}, w, z}= \begin{cases}\frac{2}{\gamma_{i}}\left[C_{i}(t)-c_{i}(t)\right], & z=1, \\
\frac{2}{\gamma_{i}}\left[\bar{C}_{i}(t)-\bar{c}_{i}(t)\right], & z=0,\end{cases}
\end{aligned}
$$


where $B_{i}(t), b_{i}(t), c_{i}(t), C_{i}(t), \bar{B}_{i}(t), \bar{b}_{i}(t), \bar{c}_{i}(t), \bar{C}_{i}(t), c_{j}(t)$, and $\bar{c}_{j}(t)$ are given by (B.18)-(B.20), (B.40)-(B.42), (B.45), and (B.47), respectively.

Proof. See Appendix B.

If $\kappa_{1}=\kappa_{2}=0$, then the model degenerates to the case where the competition factor is not considered; i.e., there is no psychological widespread between the two families; we have the following corollary.

Corollary 1. When $\kappa_{1}=\kappa_{2}=0$, the optimal time-consistent Nash equilibrium strategies for family $i(\neq j \in\{1,2\})$ are as follows:

$$
\begin{aligned}
& \tilde{p}_{i}^{*}(t)=e^{-r(T-t)}\left[\frac{r\left(\mu-r+\lambda \mu_{Y}\right)+\gamma_{i} \rho \beta \sigma\left(e^{r(T-t)}-1\right)}{\gamma_{i} r\left(\sigma^{2}+\lambda \sigma_{Y}^{2}\right)}\right], \\
& \tilde{q}_{i}^{*}(t)=e^{-r(T-t)}\left[\frac{\vartheta}{\gamma_{i} \zeta^{2} k^{\mathbb{P}}}-\frac{1}{\gamma_{i} \zeta}+\frac{c_{i 0}(t)-\bar{c}_{i 0}(t)}{\zeta}\right] 1_{\{\tau>t\}},
\end{aligned}
$$

and the equilibrium value function of family $i$ is

$$
\tilde{V}_{i}\left(t, x_{i}, w, z\right)= \begin{cases}e^{r(T-t)} x_{i}+B_{i 0}(t) w+C_{i 0}(t), & z=1, \\ e^{r(T-t)} x_{i}+\bar{B}_{i 0}(t) w+\bar{C}_{i 0}(t), & z=0 .\end{cases}
$$

Moreover, the expectation and variance of the terminal value corresponding to the equilibrium strategy for family $i$ are as follows:

$$
\begin{aligned}
\widetilde{E}_{t, x_{i}, w, z}= \begin{cases}e^{r(T-t)} x_{i}+b_{i 0}(t) w+c_{i 0}(t), & z=0, \\
e^{r(T-t)} x_{i}+\bar{b}_{i 0}(t) w+\bar{c}_{i 0}(t), & z=1,\end{cases} \\
\widetilde{\operatorname{Var}}_{t, x_{i}, w, z}= \begin{cases}\frac{2}{\gamma_{i}}\left[C_{i 0}(t)-c_{i 0}(t)\right], & z=1, \\
\frac{2}{\gamma_{i}}\left[\bar{C}_{i 0}(t)-\bar{c}_{i 0}(t)\right], & z=0,\end{cases}
\end{aligned}
$$

where

$$
\begin{aligned}
& B_{i 0}(t)=b_{i 0}(t)=\bar{B}_{i 0}(t)=\bar{b}_{i 0}(t)=-\frac{1}{r}\left(e^{r(T-t)}-1\right) \text {, } \\
& c_{i 0}(t)=-\left(\alpha-\frac{\left(\mu-r+\lambda \mu_{Y}\right) \rho \beta \sigma}{\sigma^{2}+\lambda \sigma_{Y}^{2}}\right) \frac{1}{r^{2}}\left(e^{r(T-t)}-1\right) \\
& -\left(\alpha-\frac{\left(\mu-r+\lambda \mu_{Y}\right) \rho \beta \sigma}{\sigma^{2}+\lambda \sigma_{Y}^{2}}\right) \frac{1}{r}(t-T)-\frac{\left(\mu-r+\lambda \mu_{Y}\right)^{2}}{\gamma_{i}\left(\sigma^{2}+\lambda \sigma_{Y}^{2}\right)}(t-T), \\
& C_{i 0}(t)=\left(\alpha-\frac{\left(\mu-r+\lambda \mu_{Y}\right) \rho \beta \sigma}{\sigma^{2}+\lambda \sigma_{Y}^{2}}\right)\left[-\frac{1}{r^{2}} e^{r(T-t)}-\frac{1}{r}(t-T)+\frac{1}{r^{2}}\right] \\
& +\frac{\gamma_{i} \beta^{2}}{2}\left(1-\frac{\rho^{2} \sigma^{2}}{\sigma^{2}+\lambda \sigma_{Y}^{2}}\right)\left[-\frac{1}{2 r^{3}} e^{2 r(T-t)}+\frac{2}{r^{3}} e^{r(T-t)}\right. \\
& \left.+\frac{1}{r^{2}}(t-T)+\frac{1}{2 r^{3}}-\frac{2}{r^{3}}\right]-\frac{\left(\mu-r+\lambda \mu_{Y}\right)^{2}}{2 \gamma_{i}\left(\sigma^{2}+\lambda \sigma_{Y}^{2}\right)}(t-T), \\
& \bar{c}_{i 0}(t)=e^{(\vartheta t / \zeta)} \int_{t}^{T} e^{-(\vartheta u / \zeta)}\left[\left(\alpha-\frac{\left(\mu-r+\lambda \mu_{Y}\right) \rho \beta \sigma}{\sigma^{2}+\lambda \sigma_{Y}^{2}}\right) \bar{b}_{i 0}(u)+\frac{\left(\mu-r+\lambda \mu_{Y}\right)^{2}}{\gamma_{i}\left(\sigma^{2}+\lambda \sigma_{Y}^{2}\right)}+\frac{k^{\mathbb{P}}}{\gamma_{i}}-\frac{2 \vartheta}{\gamma_{i} \zeta}+\frac{\vartheta^{2}}{\gamma_{i} \zeta^{2} k^{\mathbb{P}}}\right] \mathrm{d} u, \\
& \bar{C}_{i 0}(t)=e^{k^{\mathrm{P}} t} \int_{t}^{T} e^{-k^{\mathrm{P}} u}\left[\alpha \bar{B}_{i 0}(u)-\frac{\gamma_{i} \beta^{2}}{2}\left(1-\frac{\rho^{2} \sigma^{2}}{\sigma^{2}+\lambda \sigma_{Y}^{2}}\right) \bar{b}_{i 0}^{2}(u)\right.
\end{aligned}
$$

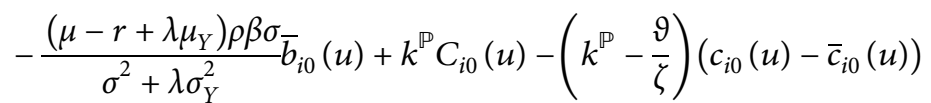

$$
\begin{aligned}
& \left.+\frac{\left(\mu-r+\lambda \mu_{Y}\right)^{2}}{2 \gamma_{i}\left(\sigma^{2}+\lambda \sigma_{Y}^{2}\right)}+\frac{k^{\mathbb{P}}}{2 \gamma_{i}}-\frac{\vartheta}{\gamma_{i} \zeta}+\frac{\vartheta^{2}}{2 \gamma_{i} \zeta^{2} k^{\mathbb{P}}}\right] \mathrm{d} u \text {. }
\end{aligned}
$$


TABLE 1: Model parameters.

\begin{tabular}{|c|c|c|c|c|c|c|c|c|c|c|c|c|c|c|c|}
\hline$r$ & $\mu$ & $\sigma$ & $\mu_{Y}$ & $\lambda$ & $\kappa_{1}$ & $\kappa_{2}$ & $\gamma_{1}$ & $\gamma_{2}$ & $\vartheta$ & $\zeta$ & $k^{\mathbb{P}}$ & $\alpha$ & $\beta$ & $\rho$ & $T$ \\
\hline 0.03 & 0.12 & 0.2 & 0.2 & 0.5 & 0.2 & 0.4 & 0.5 & 0.9 & 0.01 & 0.5 & 0.005 & 0.1 & 0.6 & -0.5 & 3 \\
\hline
\end{tabular}

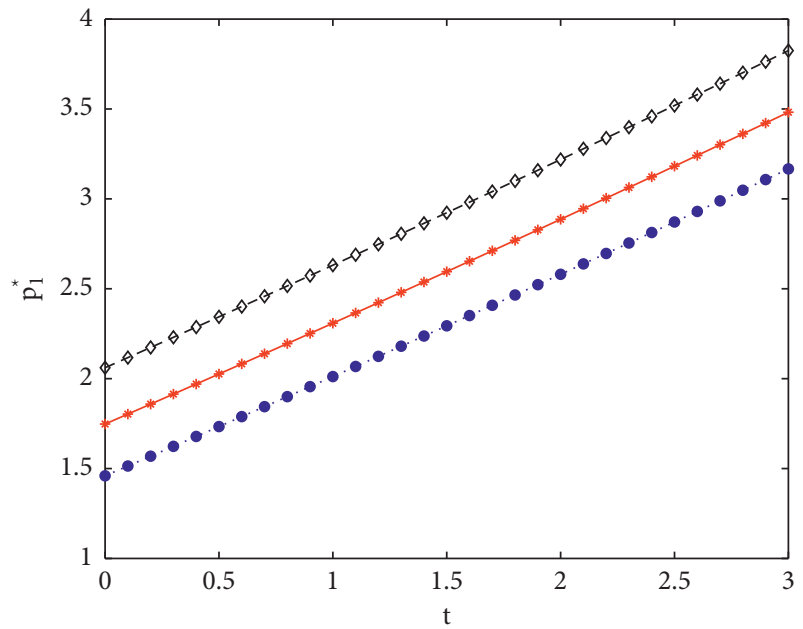

$$
\begin{array}{ll}
\cdots \bullet & \kappa_{1}=0.0 \\
-* & \kappa_{1}=0.1 \\
\text { ๑- } & \kappa_{1}=0.2
\end{array}
$$

Figure 1: The effect of $\kappa_{1}$ on $p_{1}(t)$.

\section{Remark 1}

(i) From Theorem 2, we can find that the amounts invested in risky assets $p_{i}^{*}$ and $\tilde{p}_{i}^{*}$ are influenced by the diffusion parameter $\beta$ of random expenditures

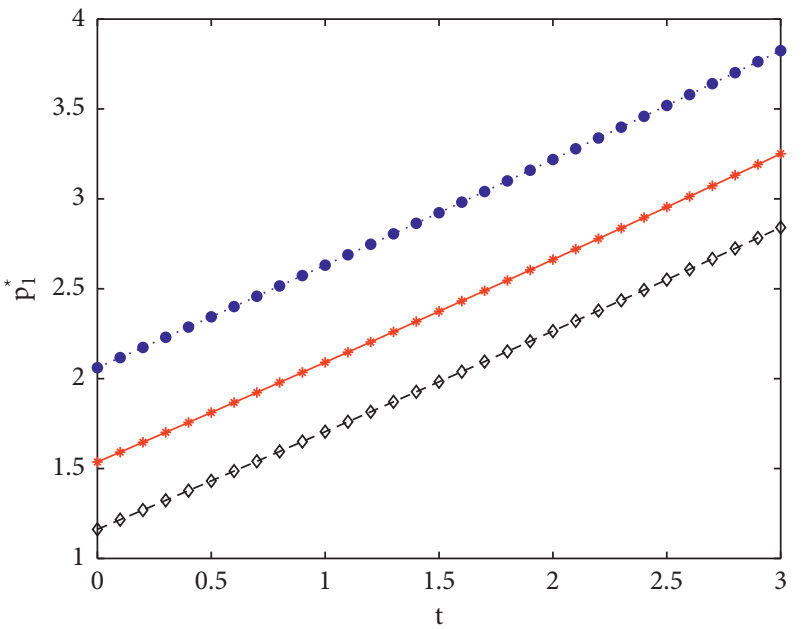

$\cdots \gamma_{1}=0.5$

$\rightarrow \gamma_{1}=0.6$

$-\diamond-\gamma_{1}=0.7$

Figure 2: The effect of $\gamma_{1}$ on $p_{1}(t)$.

but are independent of the drift parameter $\alpha$ of random expenditures. This is the same as Yuan and Lai [9]. By equations (B.16) and (B.37), we have

$$
\bar{c}_{i}^{\prime}(t)-c_{i}^{\prime}(t)-\frac{\vartheta}{\zeta}\left(\bar{c}_{i}(t)-c_{i}(t)\right)+\frac{k^{\mathbb{P}}}{\gamma_{i}}-\frac{2 \vartheta}{\gamma_{i} \zeta}+\frac{\vartheta^{2}}{\gamma_{i} \zeta^{2} k^{\mathbb{P}}}=0, \quad \bar{c}_{i}(T)-c_{i}(T)=0 .
$$

Solving the above equation and noting that $\vartheta=k^{\mathbb{Q}} \zeta$ and $(1 / \Delta)=\left(k^{\mathbb{Q}} / k^{\mathbb{P}}\right)$, we derive

$$
\bar{c}_{i}(t)-c_{i}(t)=\frac{\left(\zeta k^{\mathbb{P}}-\vartheta\right)^{2}\left(1-e^{-\vartheta(T-t) / \zeta}\right)}{\gamma_{i} \zeta \vartheta k^{\mathbb{P}}}=\frac{(\Delta-1)^{2}\left(1-e^{-\vartheta(T-t) / \zeta}\right)}{\gamma_{i} \Delta} .
$$

Symmetrically, we have

$$
\bar{c}_{j}(t)-c_{j}(t)=\frac{\left(\zeta k^{\mathbb{P}}-\vartheta\right)^{2}\left(1-e^{-\vartheta(T-t) / \zeta}\right)}{\gamma_{j} \zeta \vartheta k^{\mathbb{P}}}=\frac{(\Delta-1)^{2}\left(1-e^{-\vartheta(T-t) / \zeta}\right)}{\gamma_{j} \Delta} .
$$




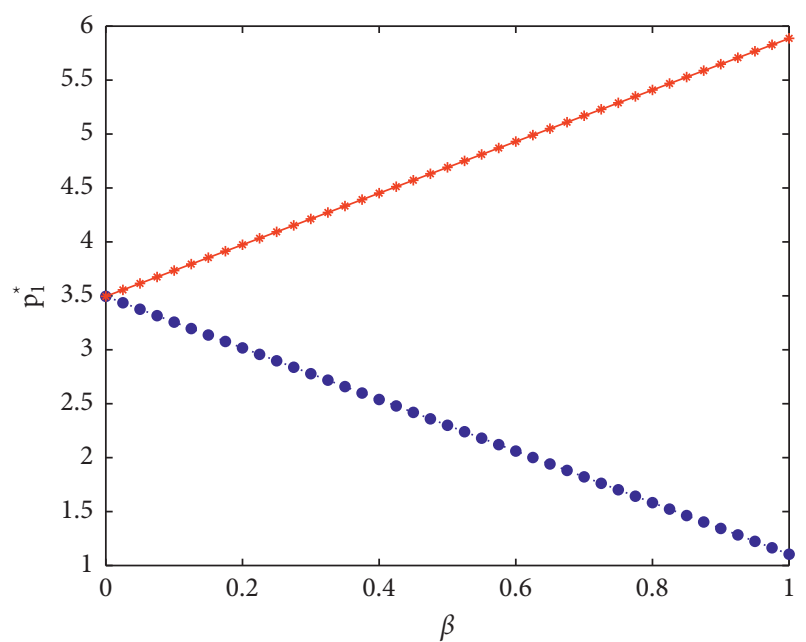

•.. $\rho=-0.5$

$*-\rho=0.5$

FIgURE 3: The effect of $\beta$ on $p_{1}(0)$.

Noting equations (17) and (21), the amount invested in defaultable bonds is easily found to be independent of random expenditures.

(ii) From equations (18), (B.18), (B.40), (22), and (24), we have

$$
\begin{aligned}
& \frac{\partial V_{i}\left(t, \hat{x}_{i}, w, z\right)}{\partial w}=-\frac{1-\kappa_{i}}{r}\left(e^{r(T-t)}-1\right)<0, \\
& \frac{\partial \widetilde{V}_{i}\left(t, x_{i}, w, z\right)}{\partial w}=-\frac{1}{r}\left(e^{r(T-t)}-1\right)<0,
\end{aligned}
$$

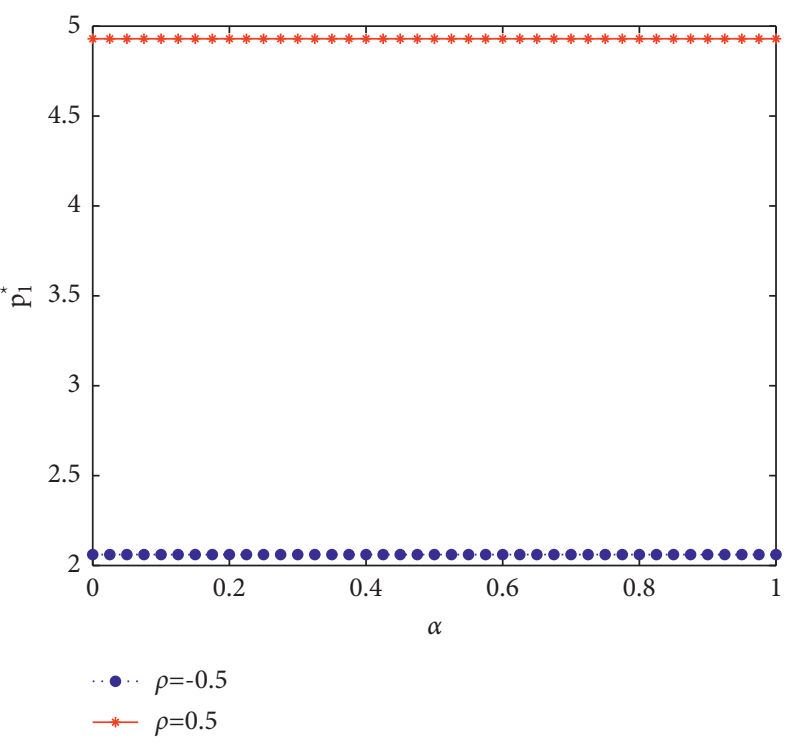

FIgURE 4: The effect of $\alpha$ on $p_{1}(0)$.

that is, expenditure brings about loss of utility.

(iii) In fact, similar to the Cournot duopoly game in classical economics, there is utility loss in the psychology of mutual comparison between the two family homes we consider. Combining Theorem 2 and Corollary 1, we define the utility loss as follows:

$$
\begin{aligned}
& \mathrm{UL}_{i}(t):= V_{i}\left(t, \hat{x}_{i}, w, z\right)-\widetilde{V}_{i}\left(t, x_{i}, w, z\right) \\
& e^{r(T-t)} \kappa_{i} x_{j}-\frac{\kappa_{i}}{r}\left(e^{r(T-t)}-1\right) w+\left(\alpha-\frac{\left(\mu-r+\lambda \mu_{Y}\right) \rho \beta \sigma}{\sigma^{2}+\lambda \sigma_{y}^{2}}\right)\left[-\frac{\kappa_{i}}{r} e^{r(T-t)}\right. \\
& \quad=\left\{\begin{array}{l}
\left.-\frac{\kappa_{i}}{r}(t-T)+\frac{\kappa_{i}}{r^{2}}\right]+\frac{\gamma_{i} \beta}{2}\left(1-\frac{\rho^{2} \sigma^{2}}{\sigma^{2}+\lambda \sigma_{Y}^{2}}\right)\left[\frac{\kappa_{i}^{2}-2 \kappa_{i}}{2 r^{3}} e^{2 r(T-t)}\right. \\
+\frac{4 \kappa_{i}-2 \kappa_{i}^{2}}{r^{3}} e^{r(T-t)}+\frac{2 \kappa_{i}-\kappa_{i}^{2}}{r^{2}}(t-T)+\frac{2 \kappa_{i}-\kappa_{i}^{2}}{\left.2 r^{3}+\frac{2 \kappa_{i}^{2}-4 \kappa_{i}}{r^{3}}\right],} z=1, \\
e^{r(T-t)} \kappa_{i} x_{j}-\frac{\kappa_{i}}{r}\left(e^{r(T-t)}-1\right) w+e^{k^{\mathbb{P}} t} \int_{t}^{T} e^{-k^{\mathbb{P}} u}\left[\alpha\left(\bar{B}_{i 0}(u)-\bar{B}_{i}(u)\right)\right. \\
-\frac{\gamma_{i} \beta^{2}}{2}\left(1-\frac{\rho^{2} \sigma^{2}}{\sigma^{2}+\lambda \sigma_{Y}^{2}}\right)\left(\bar{b}_{i 0}^{2}(u)-\bar{b}_{i}^{2}(u)\right)-\frac{\left(\mu-r+\lambda \mu_{Y}\right) \rho \beta \sigma}{\sigma^{2}+\lambda \sigma_{Y}^{2}}\left(\bar{b}_{i 0}(u)-\bar{b}_{i}(u)\right) \\
+k^{\mathbb{P}}\left(C_{i 0}(u)-C_{i}(u)\right)-\left(k^{\mathbb{P}}-\frac{\vartheta}{\zeta}\right)\left[\left(c_{i 0}(u)-\bar{c}_{i 0}(u)\right)-\left(c_{i}(u)-\bar{c}_{i}(u)\right)\right] \mathrm{d} u, \quad z=0 .
\end{array}\right.
\end{aligned}
$$




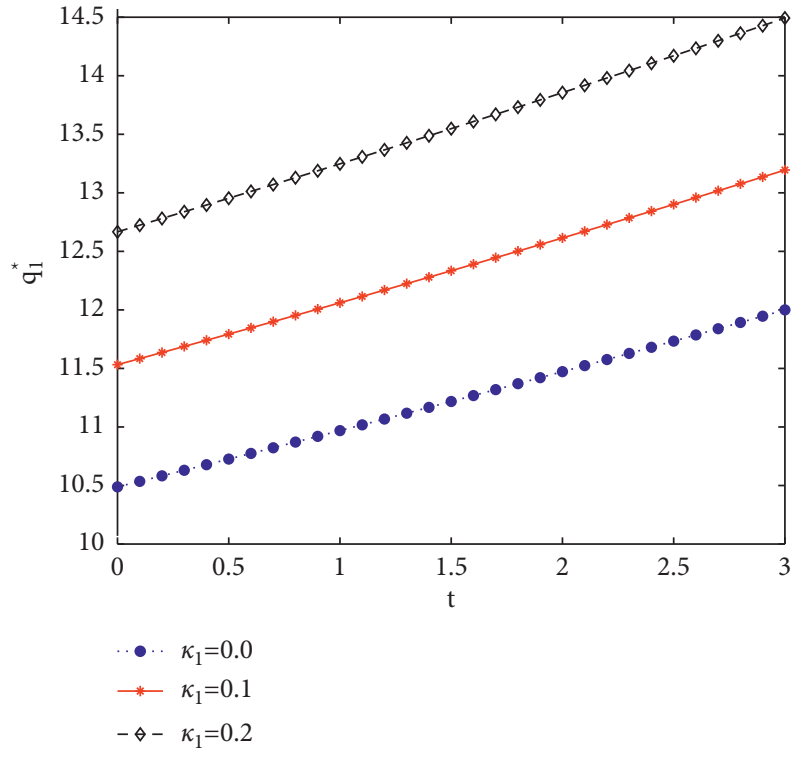

FIgURE 5: The effect of $\kappa_{1}$ on $q_{1}(t)$.

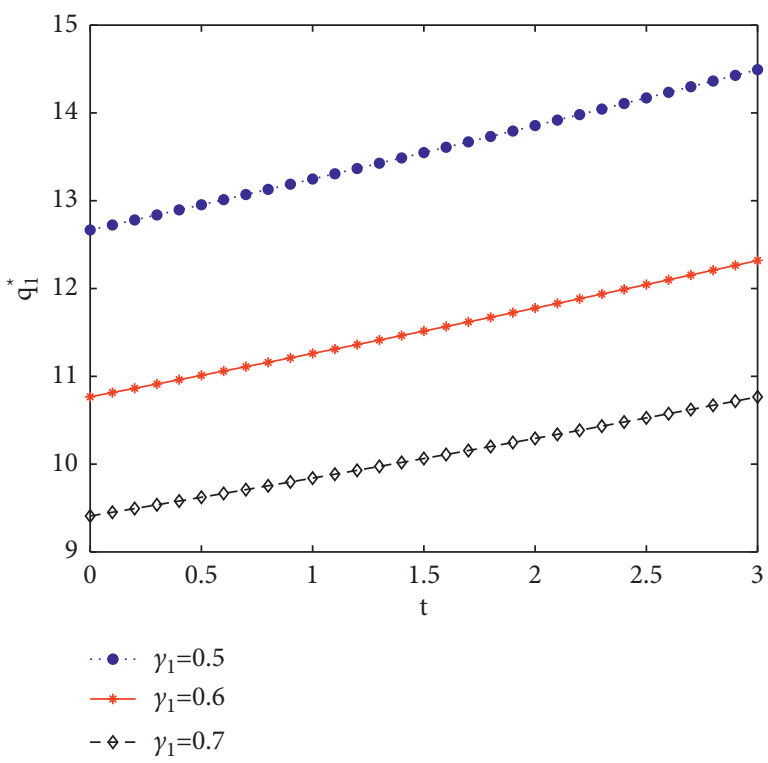

Figure 6: The effect of $\gamma_{1}$ on $q_{1}(t)$.

Notice that the utility loss $\mathrm{UL}_{i}(t) \equiv 0$ when $\kappa_{i}=0$.

\section{Sensitivity Analysis}

In this section, we provide a numerical example to illustrate the optimal time-consistent investment strategy with a random household expenditure under the relative performance, in which we analyze the effect of important parameters on results and give an economic explanation. According to the model settings in Section 2, unless otherwise stated, we select the parameters in Table 1 throughout this section. Next, we vary the value of one parameter by fixing other parameters to analyze the effect of the optimal

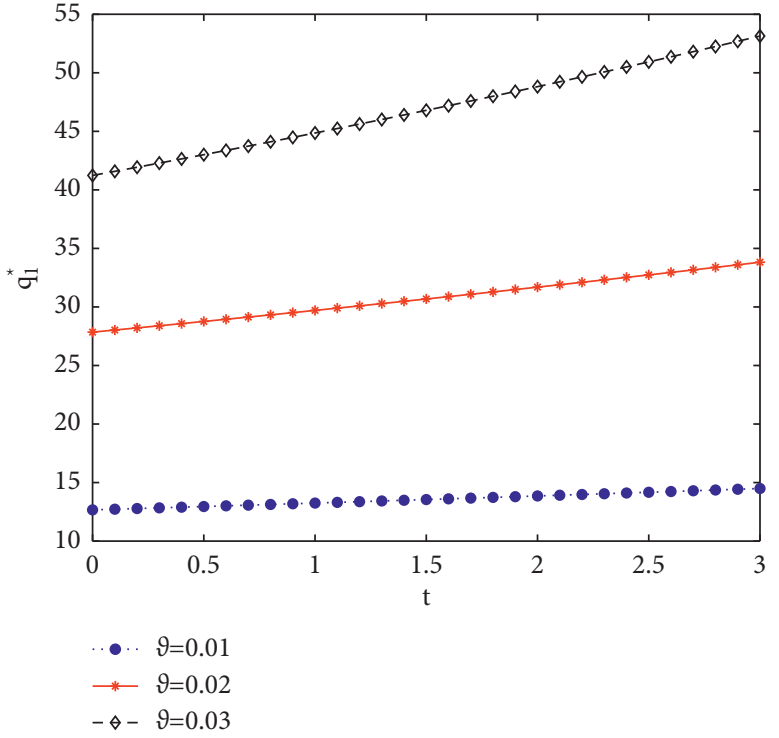

Figure 7: The effect of $\vartheta$ on $q_{1}(t)$.

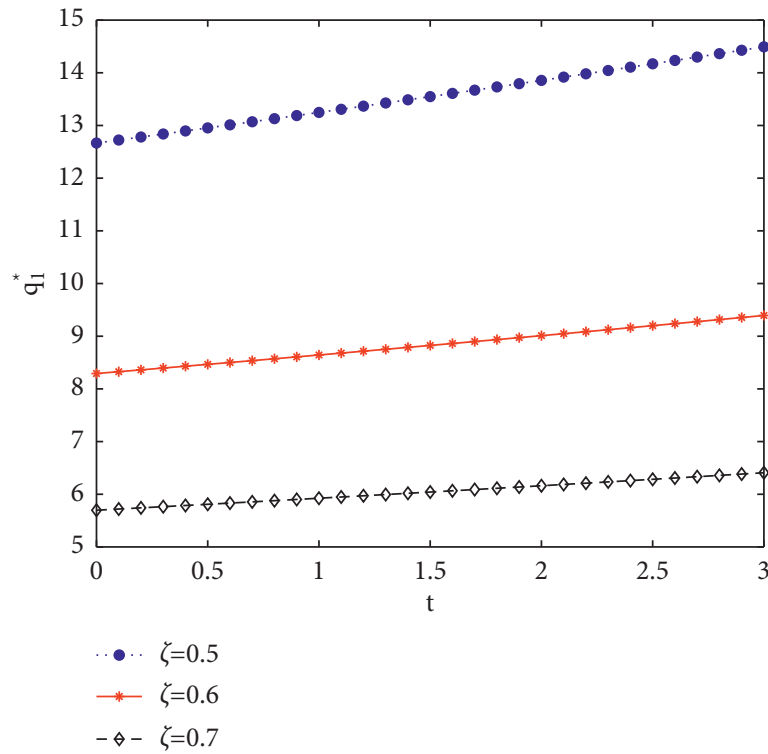

Figure 8: The effect of $\zeta$ on $q_{1}(t)$.

time-consistent strategy. Due to symmetry, the effects of the parameters have the same trend for family 1 and family 2; thus, we only analyze the effects of the parameters on the optimal results for family 1 .

Figures 1-4 depict the effects of parameters $\kappa_{1}, \gamma_{1}, \beta, \rho$, and $\alpha$ on family 1's optimal time-consistent investment strategy for risky assets, respectively. From Figure 1, it can be observed that the larger the parameter $\kappa_{1}$ that portrays the relative performance concern sensitivity, the larger the number of investments in risky assets by family 1 . This is because the larger $\kappa_{1}$ is, the greater family 1 is concerned about the comparison of the wealth of its neighbors, and if family 1 increases its exposure to risky assets, then its 


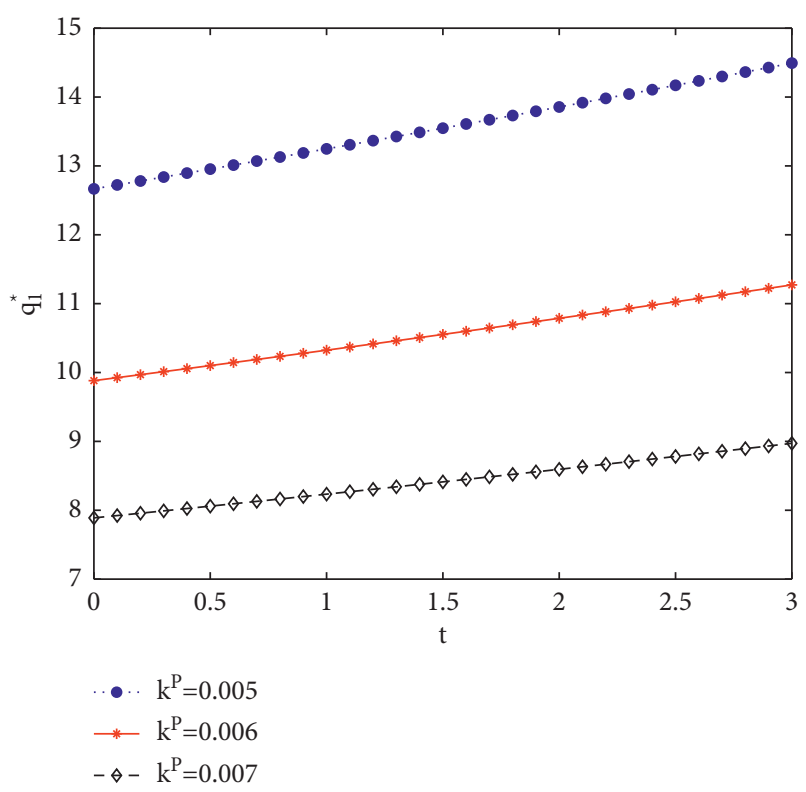

Figure 9: The effect of $k^{\mathbb{P}}$ on $q_{1}(t)$.

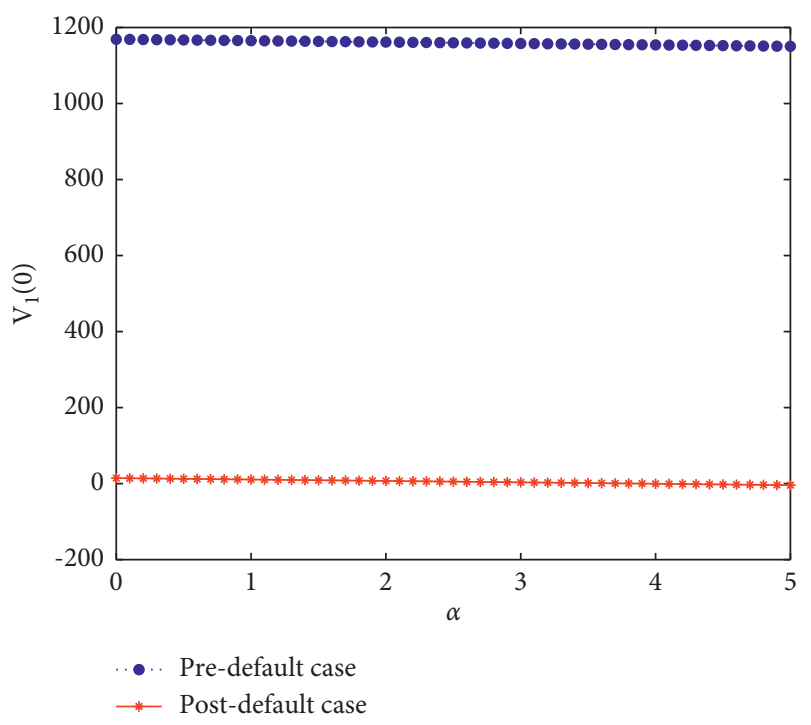

FIgURE 10: The effect of $\alpha$ on $V_{1}(0)$.

probability of creating more wealth than neighbors at the terminal moment increases. In Figure 2, the number of investments in risky assets decreases as the parameter $\gamma_{1}$ increases. $\gamma_{1}$ represents the degree of risk aversion, and a larger $\gamma_{1}$ indicates that family 1 is more risk-averse, and therefore, family 1 will reduce the number of investments in risky assets. Figure 3 shows that the amounts invested in risky assets decrease with the $\beta$ for $\rho<0$, and the amounts invested in risky assets increase with the $\beta$ for $\rho>0$. By equation (16), we have $\left(\partial p_{1}^{*} / \partial \beta\right)=\left(e^{-} r(T-t)\left(1-\kappa_{1}\right) \rho \sigma\right.$ $\left.\left(e^{r(T-t)}-1\right) /\left(1-\kappa_{1} \kappa_{2}\right) r\left(\sigma^{2}+\lambda \sigma_{Y}^{2}\right)\right) . \quad\left(\partial p_{1}^{*} / \partial \beta\right)>0 \quad$ when $\rho>0$, which means that $p_{1}^{*}$ increase with $\beta$, the family 1 is willing to invest more money in the risk asset to get more profits. In contrast, $\left(\partial p_{1}^{*} / \partial \beta\right)<0$ when $\rho<0$, that is to say, $p_{1}^{*}$ decrease with $\beta$, and the family 1 will put less money in the

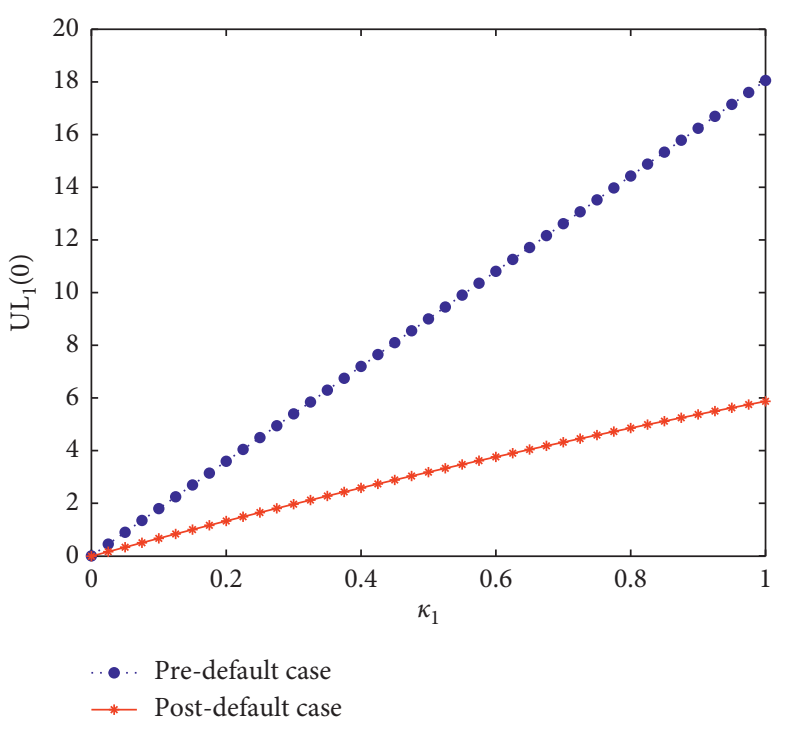

Figure 11: The effect of $\kappa_{1}$ on $\mathrm{UL}_{1}(0)$.

risky asset. From Figure 4, we can see that the amount of investments in risky assets is not affected by the average expenditure $\alpha$, which is consistent with equation (16).

Figures 5-9 illustrate the effect of parameters $\kappa_{1}, \gamma_{1}, \vartheta, \zeta$, and $k^{\mathbb{P}}$ on the amounts invested in the defaultable bonds. Figures 5 and 6 show that the amounts invested in the defaultable bonds increase with $\kappa_{1}$ and decrease with $\gamma_{1}$, in which the economic explanation is the same as Figures 1 and 2. Figures 7 and 8 show that the amounts invested in the defaultable bond $q_{1}(t)$ increase with the credit spread $\vartheta$ and decrease with the loss rate $\zeta$ because the lower credit spread $\vartheta$ or higher loss rate $\zeta$ can induce a larger potential loss. Figure 9 presents that the greater the default intensity $k^{\mathbb{P}}$, the less money will be invested in default bonds. This is because the greater the default intensity $k^{\mathbb{P}}$, the higher the default risk, and the insurer will naturally reduce the money of defaultable bonds.

Figure 10 depicts the effect of the average expenditure $\alpha$ on the equilibrium value function for family 1 and it can be found that the equilibrium value function decreases monotonically with $\alpha$, which can be interpreted as a utility loss due to the expenditure. Also, we can find that the predefault equilibrium value function is higher than the postdefault equilibrium value function, which can be interpreted as a loss of utility due to the default event. In order to portray the effect of the intensity of family 1's relative concerns parameter $\kappa_{1}$ on utility loss in Figure 11, we set $x_{2}=w=1$. From Figure 11, it can be seen that the utility loss of family 1 increases with $\kappa_{1}$; i.e., the higher the degree of mind of rivalry, the greater the utility loss. And it can be noted that when $\kappa_{1}=0$, the utility loss is equal to 0 , which is consistent with (iii) in Remark 1.

\section{Conclusion}

This paper investigates the stochastic differential game between two families for a random household expenditure with default risk under relative performance. There is a 
climbing mentality among families, especially neighbors, who will not only focus on their own wealth but also on that of their neighbors; thus, we assume that two families constitute a non-zero-sum game and that they face the same financial market consisting of a risk-free asset, a risky asset which is characterized by jump-diffusion model. And each family's random expenditure is described by Brownian motion with drift. In the mean-variance criteria and Nash equilibrium framework, by applying stochastic control theory within the framework of game theory, we obtain an explicit expression for each family's optimal time-consistent strategy and the corresponding equilibrium value function. We find that the heavier the climbing psychology, the higher the number of investments in risky assets and defaultable bonds. In addition, we define the utility loss from climbing and find that the utility loss increases with climbing psychology, similar to the static Cournot duopoly game in classical economics.

\section{Appendix}

\section{A. Proof of Theorem 1}

Suppose that the functions $V_{i}\left(t, \hat{x}_{i}, w, z\right)$ and $g_{i}\left(t, \hat{x}_{i}, w, z\right)$ satisfy the conditions of Theorem 1 . In what follows, we complete the proof in (a) and (b).

(a) We aim to show that

$$
\begin{aligned}
g_{i}\left(t, \hat{x}_{i}, w, z\right) & =E_{t, \widehat{x}_{i}, w, z}\left[\widehat{X}_{i}^{\pi_{i}^{*}, \pi_{j}^{*}}(T)\right], \\
J_{i}^{\left(\pi_{i}^{*}, \pi_{j}^{*}\right)}\left(t, \widehat{x}_{i}, w, z\right) & =V_{i}\left(t, \widehat{x}_{i}, w, z\right) .
\end{aligned}
$$

By conditions $\mathscr{L}^{\pi_{i}^{*}, \pi_{j}^{*}} g_{i}\left(t, \widehat{x}_{i}, w, z\right)=0 \quad$ and $g_{i}\left(T, \hat{x}_{i}, w, z\right)=\widehat{x}_{i}$ in Theorem 1, and by Dynkin's formula, we derive

$$
\begin{aligned}
& E_{t, \hat{x}_{i}, w, z}\left[g_{i}\left(T, \widehat{X}_{i}^{\pi_{i}^{*}, \pi_{j}^{*}}(T), w(T), z\right)\right] \\
& =g_{i}\left(t, \widehat{x}_{i}, w, z\right)+E_{t, \widehat{x}_{i}, w, z}\left[\int_{t}^{T} \mathscr{L}^{\pi_{i}^{*}, \pi_{j}^{*}} g_{i}\left(u, \widehat{X}_{i}^{\pi_{i}^{*}, \pi_{j}^{*}}(u), w(u), z\right) \mathrm{d} u\right] \\
& =g_{i}\left(t, \widehat{x}_{i}, w, z\right),
\end{aligned}
$$

where the variational operator $\mathscr{L}^{\pi_{i}, \pi_{j}}$ is defined by (14). Moreover, we obtain

$$
\begin{aligned}
g_{i}\left(t, \widehat{x}_{i}, w, z\right) & =E_{t, \widehat{x}_{i}, w, z}\left[g_{i}\left(T, \widehat{X}_{i}^{\pi_{i}^{*}, \pi_{j}^{*}}(T), w(T), z\right)\right] \\
& =E_{t, \widehat{x}_{i}, w, z}\left[\widehat{X}_{i}^{\pi_{i}^{*}, \pi_{j}^{*}}(T)\right] .
\end{aligned}
$$

Since in extended HJB equation (15), the optimal strategy for family $i$ is achieved at $\pi_{i}^{*}$, by conditions
$\mathscr{L}^{\pi_{i}^{*}, \pi_{j}^{*}} g_{i}\left(t, \hat{x}_{i}, w, z\right)=0$ and $g_{i}\left(T, \hat{x}_{i}, w, z\right)=\widehat{x}_{i}$ in Theorem 1, we rewrite extended HJB equation as $\mathscr{L}^{\pi_{i}^{*}, \pi_{j}^{*}} V_{i}\left(t, \hat{x}_{i}, w, z\right)-\frac{\gamma_{i}}{2} \mathscr{L}^{\pi_{i}^{*}, \pi_{j}^{*}} g_{i}^{2}\left(t, \hat{x}_{i}, w, z\right)=0$.

By condition $V_{i}\left(T, \hat{x}_{i}, w, z\right)=\widehat{x}_{i}$ in Theorem 1 and Dynkin's formula, we derive

$$
\begin{aligned}
E_{t, \widehat{x}_{i}, w, z}\left[\widehat{X}_{i}^{\pi_{i}^{*}, \pi_{j}^{*}}(t)\right] & =E_{t, \widehat{x}_{i}, w, z}\left[V_{i}\left(T, \widehat{X}_{i}^{\pi_{i}^{*}, \pi_{j}^{*}}(T), w(T), z\right)\right] \\
& =V_{i}\left(t, \widehat{x}_{i}, w, z\right)+E_{t, \widehat{x}_{i}, w, z}\left[\int_{t}^{T} \mathscr{L}^{\pi_{i}^{*}, \pi_{j}^{*}} V_{i}\left(u, \widehat{X}_{i}^{\pi_{i}^{*}, \pi_{j}^{*}}(u), w(u), z\right) \mathrm{d} u\right] .
\end{aligned}
$$

Inserting (A.4) into the above equation, we derive

$$
V_{i}\left(t, \widehat{x}_{i}, w, z\right)=E_{t, \widehat{x}_{i}, w, z}\left[\widehat{X}_{i}^{\pi_{i}^{*}, \pi_{j}^{*}}(t)\right]-\frac{\gamma_{i}}{2} \int_{t}^{T} \mathscr{L}^{\pi_{i}^{*}, \pi_{j}^{*}} g_{i}^{2}\left(u, \widehat{X}_{i}^{\pi_{i}^{*}, \pi_{j}^{*}}(u), w(u), z\right) \mathrm{d} u .
$$


By terminal condition and Dynkin's formula, we have

$$
\begin{aligned}
E_{t, \widehat{x}_{i}, w, z}\left[\left(\hat{X}_{i}^{\pi_{i}^{*}, \pi_{j}^{*}}(t)\right)^{2}\right] & =E_{t, \widehat{x}_{i}, w, z}\left[g_{i}^{2}\left(T, \widehat{X}_{i}^{\pi_{i}^{*}, \pi_{j}^{*}}(T), w(T), z\right)\right] \\
& =g_{i}^{2}\left(t, \widehat{x}_{i}, w, z\right)+\int_{t}^{T} \mathscr{L}^{\pi_{i}^{*}, \pi_{j}^{*}} g_{i}^{2}\left(u, \widehat{X}_{i}^{\pi_{i}^{*}, \pi_{j}^{*}}(u), w(u), z\right) \mathrm{d} u \\
& =\left(E_{t, \widehat{x}_{i}, w, z}\left[\widehat{X}_{i}^{\pi_{i}^{*}, \pi_{j}^{*}}(t)\right]\right)^{2}+\int_{t}^{T} \mathscr{L}^{\pi_{i}^{*}, \pi_{j}^{*}} g_{i}^{2}\left(u, \widehat{X}_{i}^{\pi_{i}^{*}, \pi_{j}^{*}}(u), w(u), z\right) \mathrm{d} u .
\end{aligned}
$$

That is to say, we have

$$
\operatorname{Var}_{t, \widehat{x}_{i}, w, z}\left[\widehat{X}_{i}^{\pi_{i}^{*}, \pi_{j}^{*}}(t)\right]=\int_{t}^{T} \mathscr{L}^{\pi_{i}^{*}, \pi_{j}^{*}} g_{i}^{2}\left(u, \widehat{X}_{i}^{\pi_{i}^{*}, \pi_{j}^{*}}(u), w(u), z\right) \mathrm{d} u .
$$

Putting equation (A.8) into equation (A.6), we arrive at

$$
\begin{aligned}
V_{i}\left(t, \hat{x}_{i}, w, z\right) & =E_{t, \widehat{x}_{i}, w, z}\left[\widehat{X}_{i}^{\pi_{i}^{*}, \pi_{j}^{*}}(t)\right]-\frac{\gamma_{i}}{2} \operatorname{Var}_{t, \hat{x}_{i}, w, z}\left[\widehat{X}_{i}^{\pi_{i}^{*}, \pi_{j}^{*}}(t)\right] \\
& =J_{i}^{\pi_{i}^{*}, \pi_{j}^{*}}\left(t, \widehat{x}_{i}, w, z\right) .
\end{aligned}
$$

(b) We aim to prove that $\pi_{i}^{*}$ is an equilibrium strategy of family $i$. By $J_{i}^{\pi_{i}, \pi_{j}}\left(t, \widehat{x}_{i}, w, z\right)=E_{t, \widehat{x}_{i}, w, z}\left[\widehat{X}_{i}^{\pi_{i}, \pi_{j}}(T)\right]-$ $\left(\gamma_{i} / 2\right) \operatorname{Var}_{t, \hat{x}_{i}, w, z}\left[\widehat{X}_{i}^{\pi_{i}, \pi_{j}}(T)\right]$ and the perturbed strategy $\pi_{i}^{\varepsilon}$ in Definition 2, we can derive

$$
\begin{aligned}
& J_{i}^{\pi_{i}^{\varepsilon}, \pi_{j}^{*}}\left(t, \widehat{x}_{i}, w, z\right)=E_{t, \widehat{x}_{i}, w, z}\left[\widehat{X}_{i}^{\pi_{i}^{\varepsilon}, \pi_{j}^{*}}(T)-\frac{\gamma_{i}}{2}\left(\widehat{X}_{i}^{\pi_{i}^{\varepsilon}, \pi_{j}^{*}}(T)\right)^{2}\right]+\frac{\gamma_{i}}{2}\left(E_{t, \widehat{x}_{i}, w, z}\left[\widehat{X}_{i}^{\pi_{i}^{\varepsilon}, \pi_{j}^{*}}(T)\right]\right)^{2}
\end{aligned}
$$

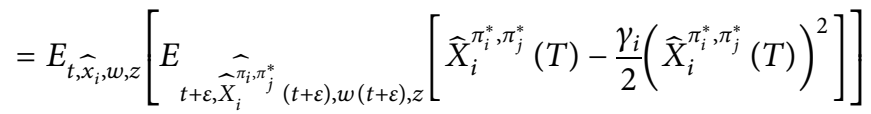

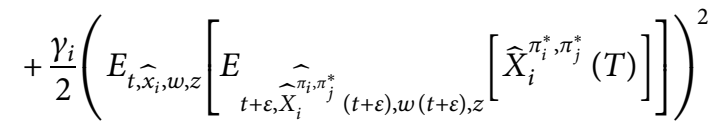

$$
\begin{aligned}
& =E_{t, \widehat{x}_{i}, w, z}\left[J_{i}^{\pi_{i}^{*}, \pi_{j}^{*}}\left(t+\varepsilon, \widehat{X}_{i}^{\hat{\pi}_{i}, \pi_{j}^{*}}(t+\varepsilon), w(t+\varepsilon), z\right)\right]
\end{aligned}
$$

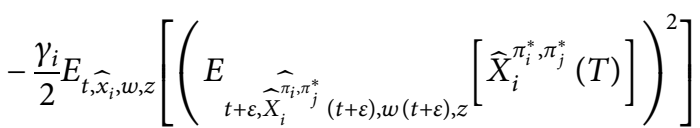


Complexity

13

$$
\begin{aligned}
& +\frac{\gamma_{i}}{2}\left(E_{t, \widehat{x}_{i}, w, z}\left[E_{t+\varepsilon, \widehat{X}_{i}^{i}, \pi_{j}^{*}(t+\varepsilon), w(t+\varepsilon), z}\left[\widehat{X}_{i}^{\pi_{i}^{*}, \pi_{j}^{*}}(T)\right]\right]\right)^{2} \\
& =E_{t, \widehat{x}_{i}, w, z}\left[J_{i}^{\pi_{i}^{*}, \pi_{j}^{*}}\left(t+\varepsilon, \widehat{X}_{i}^{\widehat{\pi}_{i}, \pi_{j}^{*}}(t+\varepsilon), w(t+\varepsilon), z\right)\right] \\
& -\frac{\gamma_{i}}{2} E_{t, \widehat{x}_{i}, w, z}\left[g_{i}^{2}\left(t+\varepsilon, \widehat{X}_{i}^{\hat{\pi}_{i}, \pi_{j}^{*}}(t+\varepsilon), w(t+\varepsilon), z\right)\right] \\
& +\frac{\gamma_{i}}{2}\left(E_{t, \widehat{x}_{i}, w, z}\left[g_{i}\left(t+\varepsilon, \widehat{X}_{i}^{\widehat{\pi}_{i}, \pi_{j}^{*}}(t+\varepsilon), w(t+\varepsilon), z\right)\right]\right)^{2} \text {, }
\end{aligned}
$$

and then we have

$$
J_{i}^{\pi_{i}^{\varepsilon}, \pi_{j}^{*}}\left(t, \widehat{x}_{i}, w, z\right)-J_{i}^{\pi_{i}^{*}, \pi_{j}^{*}}\left(t, \widehat{x}_{i}, w, z\right)=\Gamma_{\varepsilon},
$$

where

$$
\begin{aligned}
\Gamma_{\varepsilon}= & E_{t, \widehat{x}_{i}, w, z}\left[J_{i}^{\pi_{i}^{*}, \pi_{j}^{*}}\left(t+\varepsilon, \widehat{X}_{i}^{\widehat{\pi}_{i}, \pi_{j}^{*}}(t+\varepsilon), w(t+\varepsilon), z\right)\right]-J_{i}^{\pi_{i}^{*}, \pi_{j}^{*}}\left(t, \widehat{x}_{i}, w, z\right) \\
& -\frac{\gamma_{i}}{2}\left\{E_{t, \widehat{x}_{i}, w, z}\left[g_{i}^{2}\left(t+\varepsilon, \widehat{X}_{i}^{\widehat{\pi}_{i}, \pi_{j}^{*}}(t+\varepsilon), w(t+\varepsilon), z\right)\right]-g_{i}^{2}\left(t, \widehat{x}_{i}, w, z\right)\right\} \\
& +\frac{\gamma_{i}}{2}\left\{\left(E_{t, \widehat{x}_{i}, w, z}\left[g_{i}\left(t+\varepsilon, \widehat{X}_{i}^{\widehat{\pi}_{i}, \pi_{j}^{*}}(t+\varepsilon), w(t+\varepsilon), z\right)\right]\right)^{2}-g_{i}^{2}\left(t, \hat{x}_{i}, w, z\right)\right\} .
\end{aligned}
$$

For any $\pi_{i} \in \Pi_{i}$, small enough $\varepsilon>0$ and $\Phi \in C^{1,2,2}([0, T] \times \mathbb{R} \times \mathbb{R} \times\{0,1\})$, we define the following operator:

$$
\mathscr{L}_{\varepsilon}^{\pi_{i}, \pi_{j}} \Phi\left(t, \hat{x}_{i}, w, z\right)=E_{t, \widehat{x}_{i}, w, z}\left[\Phi\left(t+\varepsilon, \widehat{X}_{i}^{\widehat{\pi}_{i}, \pi_{j}^{*}}(t+\varepsilon), w(t+\varepsilon), z\right)\right]-\Phi\left(t, \widehat{x}_{i}, w, z\right)
$$

and then we have

By equations (A.12), (A.13) can be rewritten as

$$
\lim _{\varepsilon \downarrow 0}=\frac{\mathscr{L}_{\varepsilon}^{\pi_{i}, \pi_{j}} \Phi\left(t, \widehat{x}_{i}, w, z\right)}{\varepsilon}=\mathscr{L}^{\pi_{i}, \pi_{j}} \Phi\left(t, \widehat{x}_{i}, w, z\right) .
$$

$$
\begin{aligned}
\Gamma_{\varepsilon}= & \mathscr{L}_{\varepsilon}^{\widehat{\pi}_{i}, \pi_{j}^{*}} J_{i}^{\pi_{i}^{*}, \pi_{j}^{*}}\left(t, \widehat{x}_{i}, w, z\right)-\frac{\gamma_{i}}{2} \widehat{\mathscr{L}}_{\varepsilon}^{\widehat{\pi}_{i}, \pi_{j}^{*}} g_{i}^{2}\left(t, \widehat{x}_{i}, w, z\right) \\
& +\frac{\gamma_{i}}{2}\left\{\left(E_{t, \widehat{x}_{i}, w, z}\left[g_{i}\left(t+\varepsilon, \widehat{X}_{i}, \pi_{j}^{*}(t+\varepsilon), w(t+\varepsilon), z\right)\right]\right)^{2}-g_{i}^{2}\left(t, \widehat{x}_{i}, w, z\right)\right\} .
\end{aligned}
$$


By Dynkin's formula, we derive

$$
\begin{aligned}
E_{t, \widehat{x}_{i}, w, z}\left[g_{i}\left(t+\varepsilon, \widehat{X}_{i}^{\widehat{\pi}_{i}, \pi_{j}^{*}}(t+\varepsilon), w(t+\varepsilon), z\right)\right]= & g_{i}\left(t, \widehat{x}_{i}, w, z\right) \\
& +E_{t, \widehat{x}_{i}, w, z}\left[\int_{t}^{t+\varepsilon} \mathscr{L}^{\widehat{\pi}_{i}, \pi_{j}^{*}} g_{i}\left(u, \widehat{X}_{i}^{\pi_{i}, \pi_{j}^{*}}(u), w(u), z\right) \mathrm{d} u\right],
\end{aligned}
$$

which implies

$$
\begin{aligned}
& \left(E_{t, \widehat{x}_{i}, w, z}\left[g_{i}\left(t+\varepsilon, \widehat{X}_{i}^{\widehat{\pi}_{i}, \pi_{j}^{*}}(t+\varepsilon), w(t+\varepsilon), z\right)\right]\right)^{2}-g_{i}^{2}\left(t, \widehat{x}_{i}, w, z\right) \\
& =2 g_{i}\left(t, \widehat{x}_{i}, w, z\right) E_{t, \widehat{x}_{i}, w, z}\left[\int_{t}^{t+\varepsilon} \mathscr{L}^{\pi_{i}, \pi_{j}^{*}} g_{i}\left(u, \widehat{X}_{i}^{\widehat{\pi}_{i}, \pi_{j}^{*}}(u), w(u), z\right) \mathrm{d} u\right] \\
& +\left(E_{t, \widehat{x}_{i}, w, z}\left[\int_{t}^{t+\varepsilon} \widehat{\mathscr{L}}^{\widehat{\pi}_{i}, \pi_{j}^{*}} g_{i}\left(u, \widehat{X}_{i}^{\widehat{\pi}_{i}, \pi_{j}^{*}}(u), w(u), z\right) \mathrm{d} u\right]\right)^{2} .
\end{aligned}
$$

Substituting equation (A.17) into equation (A.15), we have

$$
\begin{aligned}
\Gamma_{\varepsilon}= & \mathscr{L}_{\varepsilon}^{\widehat{\pi}_{i}, \pi_{j}^{*}} J_{i}^{\pi_{i}^{*}, \pi_{j}^{*}}\left(t, \widehat{x}_{i}, w, z\right)-\frac{\gamma_{i}}{2} \widehat{L}_{\varepsilon}^{\widehat{\pi}_{i}, \pi_{j}^{*}} g_{i}^{2}\left(t, \hat{x}_{i}, w, z\right) \\
& +\gamma_{i} g_{i}\left(t, \widehat{x}_{i}, w, z\right) E_{t, \hat{x}_{i}, w, z}\left[\int_{t}^{t+\varepsilon} \mathscr{L}^{\widehat{\pi}_{i}, \pi_{j}^{*}} g_{i}\left(u, \widehat{X}_{i}^{\pi_{i}, \pi_{j}^{*}}(u), w(u), z\right) \mathrm{d} u\right] \\
& +\frac{\gamma_{i}}{2}\left(E_{t, \widehat{x}_{i}, w, z}\left[\int_{t}^{t+\varepsilon} \mathscr{L}^{\hat{\pi}_{i}, \pi_{j}^{*}} g_{i}\left(u, \widehat{X}_{i}, \pi_{j}^{*}(u), w(u), z\right) \mathrm{d} u\right]\right)^{2} .
\end{aligned}
$$

From the extended HJB equation in (15), we can obtain

$$
\begin{aligned}
& \mathscr{L}^{\widehat{T}_{i}, \pi_{j}^{*}} V_{i}\left(t, \hat{x}_{i}, w, z\right)-\frac{\gamma_{i}}{2} \mathscr{\mathscr { L }}^{\widehat{\pi}_{i}, \pi_{j}^{*}} g_{i}^{2}\left(t, \widehat{x}_{i}, w, z\right) \\
& +\gamma_{i} g_{i}\left(t, \widehat{x}_{i}, w, z\right) \mathscr{L}^{\widehat{\pi}_{i}, \pi_{j}^{*}} g_{i}\left(t, \hat{x}_{i}, w, z\right) \leq 0 .
\end{aligned}
$$

$$
\begin{aligned}
\Gamma_{\varepsilon}= & \mathscr{L}_{\varepsilon}^{\widehat{\pi}_{i}, \pi_{j}^{*}} J_{i}^{\pi_{i}^{*}, \pi_{j}^{*}}\left(t, \widehat{x}_{i}, w, z\right)-\frac{\gamma_{i}}{2} \widehat{L}_{\varepsilon}^{\widehat{\pi}_{i}, \pi_{j}^{*}} g_{i}^{2}\left(t, \widehat{x}_{i}, w, z\right) \\
& +\gamma_{i} g_{i}\left(t, \widehat{x}_{i}, w, z\right) E_{t, \widehat{x}_{i}, w, z}\left[\int_{t}^{t+\varepsilon} \widehat{\mathscr{L}}^{\widehat{\pi}_{i}, \pi_{j}^{*}} g_{i}\left(u, \widehat{X}_{i}^{\widehat{\pi}_{i}, \pi_{j}^{*}}(u), w(u), z\right) \mathrm{d} u\right] \\
& +\frac{\gamma_{i}}{2}\left(E_{t, \widehat{x}_{i}, w, z}\left[\int_{t}^{t+\varepsilon} \mathscr{L}^{\widehat{\pi}_{i}, \pi_{j}^{*}} g_{i}\left(u, \widehat{X}_{i}^{\pi_{i}, \pi_{j}^{*}}(u), w(u), z\right) \mathrm{d} u\right]\right)^{2} \leq o(\varepsilon),
\end{aligned}
$$


which means that

$$
J_{i}^{\pi_{i}^{\varepsilon}, \pi_{j}^{*}}\left(t, \widehat{x}_{i}, w, z\right)-J_{i}^{\pi_{i}^{\varepsilon}, \pi_{j}^{*}}\left(t, \widehat{x}_{i}, w, z\right)=\Gamma_{\varepsilon} \leq o(\varepsilon),
$$

i.e., $\pi_{i}^{*}$ is an equilibrium strategy for family $i$.

\section{B. Proof of Theorem 2}

For the postdefault case $(z=1)$, by the operator $\mathscr{L}^{\left(\pi_{i}, \pi_{j}\right)}$ in equation (14), the first equation in equation (15) can be rewritten as

$$
\begin{gathered}
\sup _{\pi_{i} \in \Pi_{i}}\left\{\frac{\partial V_{i}}{\partial t}+\left[r \widehat{x}_{i}+(\mu-r)\left(p_{i}(t)-\kappa_{i} p_{j}^{*}(t)\right)-\left(1-\kappa_{i}\right) w\right] \frac{\partial V_{i}}{\partial \widehat{x}_{i}}+\alpha \frac{\partial V_{i}}{\partial w}+\frac{1}{2}\left(p_{i}(t)\right.\right. \\
\left.-\kappa_{i} p_{j}^{*}(t)\right)^{2} \sigma^{2}\left[\frac{\partial^{2} V_{i}}{\partial \widehat{x}_{i}^{2}}-\gamma_{i}\left(\frac{\partial g_{i}}{\partial \widehat{x}_{i}}\right)^{2}\right]+\frac{1}{2} \beta^{2}\left[\frac{\partial^{2} V_{i}}{\partial w^{2}}-\gamma_{i}\left(\frac{\partial g_{i}}{\partial w}\right)^{2}\right]+\rho \beta \sigma\left(p_{i}(t)\right. \\
\left.-\kappa_{i} p_{j}^{*}(t)\right)\left[\frac{\partial^{2} V_{i}}{\partial \widehat{x}_{i} \partial w}-\gamma_{i} \frac{\partial g_{i}}{\partial \widehat{x}_{i}} \frac{\partial g_{i}}{\partial w}\right]+\lambda\left[E \left[V_{i}\left(t, \widehat{x}_{i}+\left(p_{i}(t)-\kappa_{i} p_{j}^{*}(t)\right) y, w, 1\right)\right.\right. \\
\left.-V_{i}\left(t, \widehat{x}_{i}, w, 1\right)\right]-\frac{\gamma_{i}}{2} E\left[g_{i}^{2}\left(t, \widehat{x}_{i}+\left(p_{i}(t)-\kappa_{i} p_{j}^{*}(t)\right) y, w, 1\right)-g_{i}^{2}\left(t, \widehat{x}_{i}, w, 1\right)\right] \\
\left.\left.+\gamma_{i} g_{i}\left(t, \widehat{x}_{i}, w, z\right) E\left[g_{i}\left(t, \widehat{x}_{i}+\left(p_{i}(t)-\kappa_{i} p_{j}^{*}(t)\right) y, w, 1\right)-g_{i}\left(t, \widehat{x}_{i}, w, 1\right)\right]\right]\right\}=0,
\end{gathered}
$$

where $V_{i}\left(T, \hat{x}_{i}, w, 1\right)=\hat{x}_{i}$ and $g_{i}\left(T, \hat{x}_{i}, w, 1\right)=\hat{x}_{i}$. Noting that the terminal conditions, we conjecture that $V_{i}$ and $g_{i}$ are of the following form:

$$
\begin{aligned}
& V_{i}\left(t, \hat{x}_{i}, w, 1\right)=A_{i}(t) \widehat{x}_{i}+B_{i}(t) w+C_{i}(t), \\
& g_{i}\left(t, \hat{x}_{i}, w, 1\right)=a_{i}(t) \widehat{x}_{i}+b_{i}(t) w+c_{i}(t),
\end{aligned}
$$

where $\quad A_{i}(T)=a_{i}(T)=1 \quad$ and $\quad B_{i}(T)=b_{i}(T)=C_{i}(T)$ $=c_{i}(T)=0$. Differentiating $V_{i}$ and $g_{i}$ with respect to $t, \widehat{x}_{i}$, and $w$, we derive

$$
\begin{aligned}
& \frac{\partial V_{i}}{\partial t}=A_{i}^{\prime}(t) \widehat{x}_{i}+B_{i}^{\prime}(t) w+C_{i}^{\prime}(t), \frac{\partial V_{i}}{\partial \widehat{x}_{i}}=A_{i}(t), \frac{\partial V_{i}}{\partial w}=B_{i}(t), \\
& \frac{\partial^{2} V_{i}}{\partial \widehat{x}_{i}^{2}}=\frac{\partial^{2} V_{i}}{\partial w^{2}}=\frac{\partial^{2} V_{i}}{\partial \widehat{x}_{i} \partial w}=0 \\
& \frac{\partial g_{i}}{\partial t}=a_{i}^{\prime}(t) \widehat{x}_{i}+b_{i}^{\prime}(t) w+c_{i}^{\prime}(t), \frac{\partial g_{i}}{\partial \widehat{x}_{i}}=a_{i}(t), \frac{\partial g_{i}}{\partial w}=b_{i}(t), \\
& \frac{\partial^{2} g_{i}}{\partial \widehat{x}_{i}^{2}}=\frac{\partial^{2} g_{i}}{\partial w^{2}}=\frac{\partial^{2} g_{i}}{\partial \widehat{x}_{i} \partial w}=0 .
\end{aligned}
$$

After calculation, we also have

$$
\begin{aligned}
E\left[V_{i}\left(t, \hat{x}_{i}+\left(p_{i}(t)-\kappa_{i} p_{j}^{*}(t)\right) y, w, 1\right)-V_{i}\left(t, \hat{x}_{i}, w, 1\right)\right]= & A_{i}(t)\left(p_{i}(t)-\kappa_{i} p_{j}^{*}(t)\right) \mu_{Y}, \\
E\left[g_{i}^{2}\left(t, \widehat{x}_{i}+\left(p_{i}(t)-\kappa_{i} p_{j}^{*}(t)\right) y, w, 1\right)-g_{i}^{2}\left(t, \widehat{x}_{i}, w, 1\right)\right]= & a_{i}^{2}(t)\left(p_{i}(t)-\kappa_{i} p_{j}^{*}(t)\right)^{2} \sigma_{Y}^{2} \\
& +2 a_{i}(t)\left(p_{i}(t)-\kappa_{i} p_{j}^{*}(t)\right) \mu_{Y}\left(a_{i}(t) \widehat{x}_{i}+b_{i}(t) w+c_{i}(t)\right), \\
E\left[g_{i}\left(t, \hat{x}_{i}+\left(p_{i}(t)-\kappa_{i} p_{j}^{*}(t)\right) y, w, 1\right)-g_{i}\left(t, \hat{x}_{i}, w, 1\right)\right]= & a_{i}(t)\left(p_{i}(t)-\kappa_{i} p_{j}^{*}(t)\right) \mu_{Y} .
\end{aligned}
$$


Substituting the above results into equation (B.1) and the second equation in equation (15), we derive

$$
\begin{aligned}
& \sup _{\pi_{i} \in \Pi_{i}}\left\{A_{i}^{\prime}(t) \widehat{x}_{i}+B_{i}^{\prime}(t) w+C_{i}^{\prime}(t)+\left[r \widehat{x}_{i}+\left(\mu-r+\lambda \mu_{Y}\right)\left(p_{i}(t)-\kappa_{i} p_{j}^{*}(t)\right)\right.\right. \\
& \left.\quad-\left(1-\kappa_{i}\right) w\right] A_{i}(t)+\alpha B_{i}(t)-\frac{\gamma_{i}}{2}\left(\sigma^{2}+\lambda \sigma_{Y}^{2}\right)\left(p_{i}(t)-\kappa_{i} p_{j}^{*}(t)\right)^{2} a_{i}^{2}(t)-\frac{\gamma_{i}}{2} \beta^{2} b_{i}^{2}(t) \\
& \left.\quad-\gamma_{i} \rho \beta \sigma\left(p_{i}(t)-\kappa_{i} p_{j}^{*}(t)\right) a_{i}(t) b_{i}(t)\right\}=0, \\
& a_{i}^{\prime}(t) \widehat{x}_{i}+b_{i}^{\prime}(t) w+c_{i}^{\prime}(t)+\left[r \widehat{x}_{i}+\left(\mu-r+\lambda \mu_{Y}\right)\left(p_{i}(t)-\kappa_{i} p_{j}^{*}(t)\right)-\left(1-\kappa_{i} w\right)\right] a_{i}(t)+\alpha b_{i}(t)=0 .
\end{aligned}
$$

By the first-order condition, we obtain and we can further obtain

$$
p_{i}^{*}(t)=\frac{\left(\mu-r+\lambda \mu_{Y}\right) A_{i}(t)}{\gamma_{i}\left(\sigma^{2}+\lambda \sigma_{Y}^{2}\right) a_{i}^{2}(t)}-\frac{\rho \beta \sigma b_{i}(t)}{\left(\sigma^{2}+\lambda \sigma_{Y}^{2}\right) a_{i}(t)}+\kappa_{i} p_{j}^{*}(t),
$$

$$
p_{i}^{*}(t)=\frac{1}{1-\kappa_{i} \kappa_{j}}\left[\frac{\left(\mu-r+\lambda \mu_{Y}\right) A_{i}(t)-\gamma_{i} \rho \beta \sigma a_{i}(t) b_{i}(t)}{\gamma_{i}\left(\sigma^{2}+\lambda \sigma_{Y}^{2}\right) a_{i}^{2}(t)}+\kappa_{i} \frac{\left(\mu-r+\lambda \mu_{Y}\right) A_{j}(t)-\gamma_{j} \rho \beta \sigma a_{j}(t) b_{j}(t)}{\gamma_{j}\left(\sigma^{2}+\lambda \sigma_{Y}^{2}\right) a_{j}^{2}(t)}\right] .
$$

Putting equation (B.8) into equations (B.6) and (B.7), we derive

$$
\begin{aligned}
& A_{i}^{\prime}(t) \widehat{x}_{i}+B_{i}^{\prime}(t) w+C_{i}^{\prime}(t)+\left[r \widehat{x}_{i}-\left(1-\kappa_{i}\right) w\right] A_{i}(t)+\alpha B_{i}(t)-\frac{\gamma_{i}}{2} \beta^{2} b_{i}^{2}(t) \\
& \quad+\frac{\left(\mu-r+\lambda \mu_{Y}\right)^{2} A_{i}^{2}(t)}{2 \gamma_{i}\left(\sigma^{2}+\lambda \sigma_{Y}^{2}\right) a_{i}^{2}(t)}-\frac{\left(\mu-r+\lambda \mu_{Y}\right) \rho \beta \sigma A_{i}(t) b_{i}(t)}{\left(\sigma^{2}+\lambda \sigma_{Y}^{2}\right) a_{i}(t)} \\
& \quad+\frac{\gamma_{i} \rho^{2} \beta^{2} \sigma^{2} b_{i}^{2}(t)}{2\left(\sigma^{2}+\lambda \sigma_{Y}^{2}\right)}=0, \\
& a_{i}^{\prime}(t) \widehat{x}_{i}+b_{i}^{\prime}(t) w+c_{i}^{\prime}(t)+\left[r \widehat{x}_{i}-\left(1-\kappa_{i}\right) w\right] a_{i}(t)+\alpha b_{i}(t) \\
& \quad+\frac{\left(\mu-r+\lambda \mu_{Y}\right)^{2} A_{i}(t)}{\gamma_{i}\left(\sigma^{2}+\lambda \sigma_{Y}^{2}\right) a_{i}(t)}-\frac{\left(\mu-r+\lambda \mu_{Y}\right) \rho \beta \sigma b_{i}(t)}{\sigma^{2}+\lambda \sigma_{Y}^{2}}=0 .
\end{aligned}
$$

By separating variables, we obtain the following differential equations:

$$
\begin{aligned}
A_{i}^{\prime}(t)+r A_{i}(t) & =0, \quad A_{i}(T)=1, \\
B_{i}^{\prime}(t)-\left(1-\kappa_{i}\right) A_{i}(t) & =0, \quad B_{i}(T)=0,
\end{aligned}
$$




$$
\begin{aligned}
& C_{i}^{\prime}(t)+\alpha B_{i}(t)-\frac{\gamma_{i}}{2} \beta^{2} b_{i}^{2}(t)+\frac{\left(\mu-r+\lambda \mu_{Y}\right)^{2} A_{i}^{2}(t)}{2 \gamma_{i}\left(\sigma^{2}+\lambda \sigma_{Y}^{2}\right) a_{i}^{2}(t)}-\frac{\left(\mu-r+\lambda \mu_{Y}\right) \rho \beta \sigma A_{i}(t) b_{i}(t)}{\left(\sigma^{2}+\lambda \sigma_{Y}^{2}\right) a_{i}(t)} \\
& \quad+\frac{\gamma_{i} \rho^{2} \beta^{2} \sigma^{2} b_{i}^{2}(t)}{2\left(\sigma^{2}+\lambda \sigma_{Y}^{2}\right)}=0, \quad C_{i}(T)=0, \\
& a_{i}^{\prime}(t)+r a_{i}(t)=0, \quad a_{i}(T)=1, \\
& b_{i}^{\prime}(t)-\left(1-\kappa_{i}\right) a_{i}(t)=0, \quad b_{i}(T)=0, \\
& c_{i}^{\prime}(t)+\alpha b_{i}(t)+\frac{\left(\mu-r+\lambda \mu_{Y}\right)^{2} A_{i}(t)}{\gamma_{i}\left(\sigma^{2}+\lambda \sigma_{Y}^{2}\right) a_{i}(t)}-\frac{\left(\mu-r+\lambda \mu_{Y}\right) \rho \beta \sigma b_{i}(t)}{\sigma^{2}+\lambda \sigma_{Y}^{2}}=0, \quad c_{i}(T)=0 .
\end{aligned}
$$

Solving the above equations, we derive

$$
\begin{aligned}
A_{i}(t)= & a_{i}(t)=e^{r(T-t)}, \\
B_{i}(t)= & b_{i}(t)=-\frac{1-\kappa_{i}}{r}\left(e^{r(T-t)}-1\right) \\
c_{i}(t)= & -\left(\alpha-\frac{\left(\mu-r+\lambda \mu_{Y}\right) \rho \beta \sigma}{\sigma^{2}+\lambda \sigma_{Y}^{2}}\right) \frac{1-\kappa_{i}}{r^{2}}\left(e^{r(T-t)}-1\right) \\
& -\left(\alpha-\frac{\left(\mu-r+\lambda \mu_{Y}\right) \rho \beta \sigma}{\sigma^{2}+\lambda \sigma_{Y}^{2}}\right) \frac{1-\kappa_{i}}{r}(t-T)-\frac{\left(\mu-r+\lambda \mu_{Y}\right)^{2}}{\gamma_{i}\left(\sigma^{2}+\lambda \sigma_{Y}^{2}\right)}(t-T), \\
C_{i}(t)= & \left(\alpha-\frac{\left(\mu-r+\lambda \mu_{Y}\right) \rho \beta \sigma}{\sigma^{2}+\lambda \sigma_{Y}^{2}}\right)\left[-\frac{1-\kappa_{i}}{r^{2}} e^{r(T-t)}-\frac{1-\kappa_{i}}{r}(t-T)+\frac{1-\kappa_{i}}{r^{2}}\right] \\
& +\frac{\gamma_{i} \beta^{2}}{2}\left(1-\frac{\rho^{2} \sigma^{2}}{\sigma^{2}+\lambda \sigma_{Y}^{2}}\right)\left[-\frac{\left(1-\kappa_{i}\right)^{2}}{2 r^{3}} e^{2 r(T-t)}+\frac{2\left(1-\kappa_{i}\right)^{2}}{r^{3}} e^{r(T-t)}\right. \\
& \left.+\frac{\left(1-\kappa_{i}\right)^{2}}{r^{2}}(t-T)+\frac{\left(1-\kappa_{i}\right)^{2}}{2 r^{3}}-\frac{2\left(1-\kappa_{i}\right)^{2}}{r^{3}}\right]-\frac{\left(\mu-r+\lambda \mu_{Y}\right)^{2}}{2 \gamma_{i}\left(\sigma^{2}+\lambda \sigma_{Y}^{2}\right)}(t-T) .
\end{aligned}
$$

Similarly, for the postdefault case $(z=0)$, the first equation in equation (15) can be rewritten as

$$
\begin{aligned}
& \sup _{\pi_{i} \in \Pi_{i}}\left\{\frac{\partial V_{i}}{\partial t}+\left[r \hat{x}_{i}+(\mu-r)\left(p_{i}(t)-\kappa_{i} p_{j}^{*}(t)\right)-\left(1-\kappa_{i}\right) w+\vartheta\left(q_{i}(t)-\kappa_{i} q_{j}(t)\right)\right] \frac{\partial V_{i}}{\partial \widehat{x}_{i}}\right. \\
& +\alpha \frac{\partial V_{i}}{\partial w}+\frac{1}{2}\left(p_{i}(t)-\kappa_{i} p_{j}^{*}(t)\right)^{2} \sigma^{2}\left[\frac{\partial^{2} V_{i}}{\partial \widehat{x}_{i}^{2}}-\gamma_{i}\left(\frac{\partial g_{i}}{\partial \widehat{x}_{i}}\right)^{2}\right]+\frac{1}{2} \beta^{2}\left[\frac{\partial^{2} V_{i}}{\partial w^{2}}-\gamma_{i}\left(\frac{\partial g_{i}}{\partial w}\right)^{2}\right] \\
& +\rho \beta \sigma\left(p_{i}(t)-\kappa_{i} p_{j}^{*}(t)\right)\left[\frac{\partial^{2} V_{i}}{\partial \widehat{x}_{i} \partial w}-\gamma_{i} \frac{\partial g_{i}}{\partial \widehat{x}_{i}} \frac{\partial g_{i}}{\partial w}\right]+\lambda\left[E \left[V_{i}\left(t, \widehat{x}_{i}+\left(p_{i}(t)-\kappa_{i} p_{j}^{*}(t)\right) y, w, 0\right)\right.\right.
\end{aligned}
$$




$$
\begin{aligned}
& \left.-V_{i}\left(t, \widehat{x}_{i}, w, 0\right)\right]-\frac{\gamma_{i}}{2} E\left[g_{i}^{2}\left(t, \hat{x}_{i}+\left(p_{i}(t)-\kappa_{i} p_{j}^{*}(t)\right) y, w, 0\right)-g_{i}^{2}\left(t, \hat{x}_{i}, w, 0\right)\right] \\
& \left.+\gamma_{i} g_{i}\left(t, \widehat{x}_{i}, w, z\right) E\left[g_{i}\left(t, \widehat{x}_{i}+\left(p_{i}(t)-\kappa_{i} p_{j}^{*}(t)\right) y, w, 0\right)-g_{i}\left(t, \widehat{x}_{i}, w, 0\right)\right]\right] \\
& +\left[V_{i}\left(t, \widehat{x}_{i}-\zeta\left(q_{i}(t)-\kappa_{i} q_{j}(t)\right), w, 1\right)-V_{i}\left(t, \widehat{x}_{i}, w, 0\right)\right] k^{\mathbb{P}} \\
& \left.-\frac{\gamma_{i}}{2}\left[g_{i}\left(t, \hat{x}_{i}-\zeta\left(q_{i}(t)-\kappa_{i} q_{j}(t)\right), w, 1\right)-g_{i}\left(t, \hat{x}_{i}, w, 0\right)\right] k^{\mathbb{P}}\right\}=0,
\end{aligned}
$$

where $V_{i}\left(T, \hat{x}_{i}, w, 0\right)=\widehat{x}_{i}$ and $g_{i}\left(T, \hat{x}_{i}, w, 0\right)=\hat{x}_{i}$. Noting the terminal conditions, we conjecture that $V_{i}$ and $g_{i}$ are of the following form:

$$
\begin{gathered}
V_{i}\left(t, \hat{x}_{i}, w, 0\right)=\bar{A}_{i}(t) \hat{x}_{i}+\bar{B}_{i}(t) w+\bar{C}_{i}(t), \\
g_{i}\left(t, \hat{x}_{i}, w, 0\right)=\bar{a}_{i}(t) \hat{x}_{i}+\bar{b}_{i}(t) w+\bar{c}_{i}(t),
\end{gathered}
$$

where $\bar{A}_{i}(T)=\bar{a}_{i}(T)=1$ and $\bar{B}_{i}(T)=\bar{b}_{i}(T)=\bar{C}_{i}(T)=$ $\bar{c}_{i}(T)=0$. Differentiating $V_{i}$ and $g_{i}$ with respect to $t, \widehat{x}_{i}$, and $w$, we derive

$$
\begin{aligned}
\frac{\partial V_{i}}{\partial t} & =\bar{A}_{i}^{\prime}(t) \widehat{x}_{i}+\bar{B}_{i}^{\prime}(t) w+\bar{C}_{i}^{\prime}(t), \frac{\partial V_{i}}{\partial \widehat{x}_{i}}=\bar{A}_{i}(t), \frac{\partial V_{i}}{\partial w}=\bar{B}_{i}(t) \\
\frac{\partial^{2} V_{i}}{\partial \widehat{x}_{i}^{2}} & =\frac{\partial^{2} V_{i}}{\partial w^{2}}=\frac{\partial^{2} V_{i}}{\partial \widehat{x}_{i} \partial w}=0 \\
\frac{\partial g_{i}}{\partial t} & =\bar{a}_{i}^{\prime}(t) \widehat{x}_{i}+\bar{b}_{i}^{\prime}(t) w+\bar{c}_{i}^{\prime}(t), \frac{\partial g_{i}}{\partial \widehat{x}_{i}}=\bar{a}_{i}(t), \frac{\partial g_{i}}{\partial w}=\bar{b}_{i}(t), \\
\frac{\partial^{2} g_{i}}{\partial \widehat{x}_{i}^{2}} & =\frac{\partial^{2} g_{i}}{\partial w^{2}}=\frac{\partial^{2} g_{i}}{\partial \widehat{x}_{i} \partial w}=0
\end{aligned}
$$

After calculation, we also have

$$
\begin{aligned}
E\left[V_{i}\left(t, \hat{x}_{i}+\left(p_{i}(t)-\kappa_{i} p_{j}^{*}(t)\right) y, w, 0\right)-V_{i}\left(t, \hat{x}_{i}, w, 0\right)\right]= & \bar{A}_{i}(t)\left(p_{i}(t)-\kappa_{i} p_{j}^{*}(t)\right) \mu_{Y}, \\
E\left[g_{i}^{2}\left(t, \hat{x}_{i}+\left(p_{i}(t)-\kappa_{i} p_{j}^{*}(t)\right) y, w, 0\right)-g_{i}^{2}\left(t, \hat{x}_{i}, w, 0\right)\right]= & \bar{a}_{i}^{2}(t)\left(p_{i}(t)-\kappa_{i} p_{j}^{*}(t)\right)^{2} \sigma_{Y}^{2} \\
& +2 \bar{a}_{i}(t)\left(p_{i}(t)-\kappa_{i} p_{j}^{*}(t)\right) \mu_{Y}\left(\bar{a}_{i}(t) \hat{x}_{i}+\bar{b}_{i}(t) w+\bar{c}_{i}(t)\right), \\
E\left[g_{i}\left(t, \hat{x}_{i}+\left(p_{i}(t)-\kappa_{i} p_{j}^{*}(t)\right) y, w, 0\right)-g_{i}\left(t, \hat{x}_{i}, w, 0\right)\right]= & \bar{a}_{i}(t)\left(p_{i}(t)-\kappa_{i} p_{j}^{*}(t)\right) \mu_{Y}, \\
V_{i}\left(t, \hat{x}_{i}-\zeta\left(q_{i}(t)-\kappa_{i} q_{j}(t)\right), w, 1\right)-V_{i}\left(t, \hat{x}_{i}, w, 0\right)= & A_{i}(t)\left(\hat{x}_{i}-\zeta\left(q_{i}(t)-\kappa_{i} q_{j}(t)\right)\right) \\
& -\bar{A}_{i} \hat{x}_{i}+\left(B_{i}(t)-\bar{B}_{i}(t)\right) w+C_{i}(t)-\bar{C}_{i}(t), \\
g_{i}\left(t, \hat{x}_{i}-\zeta\left(q_{i}(t)-\kappa_{i} q_{j}(t)\right), w, 1\right)-g_{i}\left(t, \hat{x}_{i}, w, 0\right)= & a_{i}(t)\left(\hat{x}_{i}-\zeta\left(q_{i}(t)-\kappa_{i} q_{j}(t)\right)\right) \\
& -\bar{a}_{i} \hat{x}_{i}+\left(b_{i}(t)-\bar{b}_{i}(t)\right) w+c_{i}(t)-\bar{c}_{i}(t) .
\end{aligned}
$$


Inserting the above results into equation (B.1) and the second equation in equation (15), we obtain

$$
\begin{aligned}
& \sup _{\pi_{i} \in \Pi_{i}}\left\{\bar{A}_{i}^{\prime}(t) \widehat{x}_{i}+\bar{B}_{i}^{\prime}(t) w+\bar{C}_{i}^{\prime}(t)+\left[r \widehat{x}_{i}+\left(\mu-r+\lambda \mu_{Y}\right)\left(p_{i}(t)-\kappa_{i} p_{j}^{*}(t)\right)+\vartheta\left(q_{i}(t)\right.\right.\right. \\
& \left.\left.-\kappa_{i} q_{j}^{*}(t)\right)-\left(1-\kappa_{i}\right) w\right] \bar{A}_{i}(t)+\alpha \bar{B}_{i}(t)-\frac{\gamma_{i}}{2}\left(\sigma^{2}+\lambda \sigma_{Y}^{2}\right)\left(p_{i}(t)-\kappa_{i} p_{j}^{*}(t)\right)^{2} \bar{a}_{i}^{2}(t) \\
& \quad-\frac{\gamma_{i}}{2} \beta^{2} \bar{b}_{i}^{2}(t)-\gamma_{i} \rho \beta \sigma\left(p_{i}(t)-\kappa_{i} p_{j}^{*}(t)\right) \bar{a}_{i}(t) \bar{b}_{i}(t)+\left[A _ { i } ( t ) \left(\left(\hat{x}_{i}-\zeta\left(q_{i}(t)-\kappa_{i} q_{j}^{*}(t)\right)\right)\right.\right. \\
& \left.-\bar{A}_{i} \hat{x}_{i}+\left(B_{i}(t)-\bar{B}_{i}(t)\right) w+C_{i}(t)-\bar{C}_{i}(t)\right] k^{\mathbb{P}}-\frac{\gamma_{i}}{2}\left[a_{i}(t)\left(\hat{x}_{i}-\zeta\left(q_{i}(t)-\kappa_{i} q_{j}^{*}(t)\right)\right)\right. \\
& \left.\left.-\bar{a}_{i} \widehat{x}_{i}+\left(b_{i}(t)-\bar{b}_{i}(t)\right) w+c_{i}(t)-\bar{c}_{i}(t)\right]^{2} k^{\mathbb{P}}\right\}=0, \\
& \bar{a}_{i}^{\prime}(t) \hat{x}_{i}+\bar{b}_{i}^{\prime}(t) w+\bar{c}_{i}^{\prime}(t)+\left[r \hat{x}_{i}+\left(\mu-r+\lambda \mu_{Y}\right)\left(p_{i}^{*}(t)-\kappa_{i} p_{j}^{*}(t)\right)-\left(1-\kappa_{i} w\right)\right. \\
& \left.+\vartheta\left(q_{i}^{*}(t)-\kappa_{i} q_{j}^{*}(t)\right)\right] \bar{a}_{i}(t)+\alpha \bar{b}_{i}(t)+\left[a_{i}(t)\left(\widehat{x}_{i}-\zeta\left(q_{i}^{*}(t)-\kappa_{i} q_{j}^{*}(t)\right)\right)-\bar{a}_{i} \widehat{x}_{i}\right. \\
& \left.+\left(b_{i}(t)-\bar{b}_{i}(t)\right) w+c_{i}(t)-\bar{c}_{i}(t)\right] k^{\mathbb{P}}=0 .
\end{aligned}
$$

Applying again the first-order condition, we derive

$$
\begin{aligned}
& p_{i}^{*}(t)=\frac{\left(\mu-r+\lambda \mu_{Y}\right) \bar{A}_{i}(t)}{\gamma_{i}\left(\sigma^{2}+\lambda \sigma_{Y}^{2}\right) \bar{a}_{i}^{2}(t)}-\frac{\rho \beta \sigma \bar{b}_{i}(t)}{\left(\sigma^{2}+\lambda \sigma_{Y}^{2}\right) \bar{a}_{i}(t)}+\kappa_{i} p_{j}^{*}(t), \\
& q_{i}^{*}(t)=\frac{\vartheta \bar{A}_{i}(t)}{\gamma_{i} \zeta^{2} k^{\mathbb{P}} a_{i}^{2}(t)}-\frac{A_{i}(t)}{\gamma_{i} \zeta a_{i}^{2}(t)}+\frac{\widehat{x}_{i}}{\zeta}-\frac{\bar{a}_{i}(t) \widehat{x}_{i}}{\zeta a_{i}(t)}+\frac{\left(b_{i}(t)-\bar{b}_{i}(t)\right) w}{\zeta a_{i}(t)}+\frac{c_{i}(t)-\bar{c}_{i}(t)}{\zeta a_{i}(t)}+\kappa_{i} q_{j}^{*}(t),
\end{aligned}
$$

and we can also derive

$$
\begin{aligned}
p_{i}^{*}(t)= & \frac{1}{1-\kappa_{i} \kappa_{j}}\left[\frac{\left(\mu-r+\lambda \mu_{Y}\right) \bar{A}_{i}(t)-\gamma_{i} \rho \beta \sigma \bar{a}_{i}(t) \bar{b}_{i}(t)}{\gamma_{i}\left(\sigma^{2}+\lambda \sigma_{Y}^{2}\right) \bar{a}_{i}^{2}(t)}+\kappa_{i} \frac{\left(\mu-r+\lambda \mu_{Y}\right) \bar{A}_{j}(t)-\gamma_{j} \rho \beta \sigma \bar{a}_{j}(t) \bar{b}_{j}(t)}{\gamma_{j}\left(\sigma^{2}+\lambda \sigma_{Y}^{2}\right) \bar{a}_{j}^{2}(t)}\right], \\
q_{i}^{*}(t)= & \frac{1}{1-\kappa_{i} \kappa_{j}}\left[\frac{\vartheta \bar{A}_{i}(t)}{\gamma_{i} \zeta^{2} k^{\mathbb{P}} a_{i}^{2}(t)}-\frac{A_{i}(t)}{\gamma_{i} \zeta a_{i}^{2}(t)}+\frac{\widehat{x}_{i}}{\zeta}-\frac{\bar{a}_{i}(t) \widehat{x}_{i}}{\zeta a_{i}(t)}+\frac{\left(b_{i}(t)-\bar{b}_{i}(t)\right) w}{\zeta a_{i}(t)}\right. \\
& +\frac{c_{i}(t)-\bar{c}_{i}(t)}{\zeta a_{i}(t)}+\kappa_{i}\left(\frac{\vartheta \bar{A}_{j}(t)}{\gamma_{j} \zeta^{2} k^{\mathbb{P}} a_{j}^{2}(t)}-\frac{A_{j}(t)}{\gamma_{j} \zeta a_{j}^{2}(t)}+\frac{\hat{x}_{j}}{\zeta}-\frac{\bar{a}_{j}(t) \hat{x}_{j}}{\zeta a_{j}(t)}\right. \\
& \left.\left.+\frac{\left(b_{j}(t)-\bar{b}_{j}(t)\right) w}{\zeta a_{j}(t)}+\frac{c_{j}(t)-\bar{c}_{j}(t)}{\zeta a_{j}(t)}\right)\right] .
\end{aligned}
$$


Putting equations (B.28) and (B.29) into equations (B.26) and (B.27), we derive

$$
\begin{aligned}
& \bar{A}_{i}^{\prime}(t) \widehat{x}_{i}+\bar{B}_{i}^{\prime}(t) w+\bar{C}_{i}^{\prime}(t)+\left[r \widehat{x}_{i}-\left(1-\kappa_{i}\right) w\right] \bar{A}_{i}(t)+\alpha \bar{B}_{i}(t)-\frac{\gamma_{i}}{2} \beta^{2} \bar{b}_{i}^{2}(t) \\
& +\frac{\left(\mu-r+\lambda \mu_{Y}\right)^{2} \bar{A}_{i}^{2}(t)}{2 \gamma_{i}\left(\sigma^{2}+\lambda \sigma_{Y}^{2}\right) \bar{a}_{i}^{2}(t)}-\frac{\left(\mu-r+\lambda \mu_{Y}\right) \rho \beta \sigma \bar{A}_{i}(t) \bar{b}_{i}(t)}{\left(\sigma^{2}+\lambda \sigma_{Y}^{2}\right) \bar{a}_{i}(t)} \\
& +\frac{\gamma_{i} \rho^{2} \beta^{2} \sigma^{2} \bar{b}_{i}^{2}(t)}{2\left(\sigma^{2}+\lambda \sigma_{Y}^{2}\right)}+\left[\frac{\vartheta \bar{A}_{i}(t)}{\zeta}-\frac{\vartheta \bar{A}_{i}(t) \bar{a}_{i}(t)}{\zeta a_{i}(t)}-k^{\mathbb{P}} \bar{A}_{i}(t)+\frac{k^{\mathbb{P}} A_{i}(t) \bar{a}_{i}(t)}{a_{i}(t)}\right] \widehat{x}_{i} \\
& +\left[\frac{\vartheta \bar{A}_{i}(t)\left(b_{i}(t)-\bar{b}_{i}(t)\right)}{\zeta a_{i}(t)}-\frac{k^{\mathbb{P}} A_{i}(t)\left(b_{i}(t)-\bar{b}_{i}(t)\right)}{a_{i}(t)}+k^{\mathbb{P}}\left(B_{i}(t)-\bar{B}_{i}(t)\right)\right] w \\
& +k^{\mathbb{P}}\left(C_{i}(t)-\bar{C}_{i}(t)\right)-\frac{k^{\mathbb{P}} A_{i}(t)\left(c_{i}(t)-\bar{c}_{i}(t)\right)}{a_{i}(t)}+\frac{\vartheta \bar{A}_{i}(t)\left(c_{i}(t)-\bar{c}_{i}(t)\right)}{\zeta a_{i}(t)} \\
& +\frac{k^{\mathbb{P}} A_{i}^{2}(t)}{2 \gamma_{i} a_{i}^{2}(t)}-\frac{\vartheta A_{i}(t) \bar{A}_{i}(t)}{\gamma_{i} \zeta a_{i}^{2}(t)}+\frac{\vartheta^{2} \bar{A}_{i}^{2}(t)}{2 \gamma_{i} \zeta^{2} k^{\mathbb{P}} a_{i}^{2}(t)}=0, \\
& \bar{a}_{i}^{\prime}(t) \widehat{x}_{i}+\bar{b}_{i}^{\prime}(t) w+\bar{c}_{i}^{\prime}(t)+\left[r \widehat{x}_{i}-\left(1-\kappa_{i}\right) w\right] \bar{a}_{i}(t)+\alpha \bar{b}_{i}(t)+\frac{\left(\mu-r+\lambda \mu_{Y}\right)^{2} \bar{A}_{i}(t)}{\gamma_{i}\left(\sigma^{2}+\lambda \sigma_{Y}^{2}\right) \bar{a}_{i}(t)} \\
& -\frac{\left(\mu-r+\lambda \mu_{Y}\right) \rho \beta \sigma \bar{b}_{i}(t)}{\sigma^{2}+\lambda \sigma_{Y}^{2}}+\left[\frac{\vartheta \bar{a}_{i}(t)}{\zeta}-\frac{\vartheta \bar{a}_{i}^{2}(t)}{\zeta a_{i}(t)}\right] \widehat{x}_{i}+\frac{\vartheta \bar{a}_{i}(t)\left(b_{i}(t)-\bar{b}_{i}(t)\right)}{\zeta a_{i}(t)} w \\
& +\frac{k^{\mathbb{P}} A_{i}(t)}{\gamma_{i} a_{i}(t)}-\frac{\vartheta \bar{A}_{i}(t)}{\gamma_{i} \zeta a_{i}(t)}-\frac{\vartheta A_{i}(t) \bar{a}_{i}(t)}{\gamma_{i} \zeta a_{i}^{2}(t)}+\frac{\vartheta^{2} \bar{A}_{i}(t) \bar{a}_{i}(t)}{\gamma_{i} \zeta^{2} k^{\mathbb{P}} a_{i}^{2}(t)} \\
& +\frac{\vartheta \bar{a}_{i}(t)\left(c_{i}(t)-\bar{c}_{i}(t)\right)}{\zeta a_{i}(t)}=0
\end{aligned}
$$

By separating variables, we obtain the following differential equations:

$$
\begin{aligned}
& \bar{A}_{i}^{\prime}(t)+\left(r+\frac{\vartheta}{\zeta}-k^{\mathbb{P}}\right) \bar{A}_{i}(t)-\left(\frac{\vartheta \bar{A}_{i}(t)}{\zeta a_{i}(t)}-k^{\mathbb{P}}\right) \bar{a}_{i}(t)=0, \quad \bar{A}_{i}(T)=1, \\
& \bar{a}_{i}^{\prime}(t)+\left(r+\frac{\vartheta}{\zeta}\right) \bar{a}_{i}(t)-\frac{\vartheta \bar{a}_{i}^{2}(t)}{\zeta a_{i}(t)}=0, \quad \bar{a}_{i}(T)=1, \\
& \bar{b}_{i}^{\prime}(t)-\frac{\vartheta \bar{a}_{i}(t)}{\zeta a_{i}(t)} \bar{b}_{i}(t)-\left(1-\kappa_{i}\right) \bar{a}_{i}(t)+\frac{\vartheta \bar{a}_{i}(t) b_{i}(t)}{\zeta a_{i}(t)}=0, \quad \bar{b}_{i}(T)=0, \\
& \bar{B}_{i}^{\prime}(t)-k^{\mathbb{P}} \bar{B}_{i}(t)-\left(1-\kappa_{i}\right) \bar{A}_{i}(t)+k^{\mathbb{P}} B_{i}(t)+\frac{\vartheta \bar{A}_{i}(t)\left(b_{i}(t)-\bar{b}_{i}(t)\right)}{\zeta a_{i}(t)} \\
& -\frac{k^{\mathbb{P}} A_{i}(t)\left(b_{i}(t)-\bar{b}_{i}(t)\right)}{a_{i}(t)}=0, \quad \bar{B}_{i}(T)=0,
\end{aligned}
$$




$$
\begin{aligned}
& \bar{c}_{i}^{\prime}(t)-\frac{\vartheta \bar{a}_{i}(t)}{\zeta a_{i}(t)} \bar{c}_{i}(t)+\alpha \bar{b}_{i}(t)+\frac{\left(\mu-r+\lambda \mu_{Y}\right)^{2} \bar{A}_{i}(t)}{\gamma_{i}\left(\sigma^{2}+\lambda \sigma_{Y}^{2}\right) \bar{a}_{i}(t)}-\frac{\left(\mu-r+\lambda \mu_{Y}\right) \rho \beta \sigma \bar{b}_{i}(t)}{\sigma^{2}+\lambda \sigma_{Y}^{2}} \\
& +\frac{k^{\mathbb{P}} A_{i}(t)}{\gamma_{i} a_{i}(t)}-\frac{\vartheta \bar{A}_{i}(t)}{\gamma_{i} \zeta a_{i}(t)}-\frac{\vartheta A_{i}(t) \bar{a}_{i}(t)}{\gamma_{i} \zeta a_{i}^{2}(t)}+\frac{\vartheta^{2} \bar{A}_{i}(t) \bar{a}_{i}(t)}{\gamma_{i} \zeta^{2} k^{\mathbb{P}} a_{i}^{2}(t)} \\
& +\frac{\vartheta \bar{a}_{i}(t) c_{i}(t)}{\zeta a_{i}(t)}=0, \quad \bar{c}_{i}(T)=0, \\
& \bar{C}_{i}^{\prime}(t)-k^{\mathbb{P}} \bar{C}_{i}(t)+\alpha \bar{B}_{i}(t)-\frac{\gamma_{i}}{2} \beta^{2} \bar{b}_{i}^{2}(t)+\frac{\left(\mu-r+\lambda \mu_{Y}\right)^{2} \bar{A}_{i}^{2}(t)}{2 \gamma_{i}\left(\sigma^{2}+\lambda \sigma_{Y}^{2}\right) \bar{a}_{i}^{2}(t)} \\
& -\frac{\left(\mu-r+\lambda \mu_{Y}\right) \rho \beta \sigma \bar{A}_{i}(t) \bar{b}_{i}(t)}{\left(\sigma^{2}+\lambda \sigma_{Y}^{2}\right) \bar{a}_{i}(t)}+\frac{\gamma_{i} \rho^{2} \beta^{2} \sigma^{2} \bar{b}_{i}^{2}(t)}{2\left(\sigma^{2}+\lambda \sigma_{Y}^{2}\right)}+k^{\mathbb{P}} C_{i}(t) \\
& -\frac{k^{\mathbb{P}} A_{i}(t)\left(c_{i}(t)-\bar{c}_{i}(t)\right)}{a_{i}(t)}+\frac{\vartheta \bar{A}_{i}(t)\left(c_{i}(t)-\bar{c}_{i}(t)\right)}{\zeta a_{i}(t)}+\frac{k^{\mathbb{P}} A_{i}^{2}(t)}{2 \gamma_{i} a_{i}^{2}(t)}-\frac{\vartheta A_{i}(t) \bar{A}_{i}(t)}{\gamma_{i} \zeta a_{i}^{2}(t)} \\
& +\frac{\vartheta^{2} \bar{A}_{i}^{2}(t)}{2 \gamma_{i} \zeta^{2} k^{\mathbb{P}} a_{i}^{2}(t)}=0, \quad \bar{C}_{i}(T)=0 .
\end{aligned}
$$

Solving the above equations, we derive

$$
\begin{aligned}
\bar{A}_{i}(t)= & \bar{a}_{i}(t)=e^{r(T-t)}, \\
\bar{B}_{i}(t)= & \bar{b}_{i}(t)=-\frac{1-\kappa_{i}}{r}\left(e^{r(T-t)}-1\right), \\
\bar{c}_{i}(t)= & e^{(\vartheta t / \zeta)} \int_{t}^{T} e^{-(\vartheta t / \zeta)}\left[\left(\alpha-\frac{\left(\mu-r+\lambda \mu_{Y}\right) \rho \beta \sigma}{\sigma^{2}+\lambda \sigma_{Y}^{2}}\right) \bar{b}_{i}(u)+\frac{\left(\mu-r+\lambda \mu_{Y}\right)^{2}}{\gamma_{i}\left(\sigma^{2}+\lambda \sigma_{Y}^{2}\right)}+\frac{k^{\mathbb{P}}}{\gamma_{i}}-\frac{2 \vartheta}{\gamma_{i} \zeta}+\frac{\vartheta^{2}}{\gamma_{i} \zeta^{2} k^{\mathbb{P}}}\right] \mathrm{d} u, \\
\bar{C}_{i}(t)= & e^{k^{\mathbb{P} t}} \int_{t}^{T} e^{-k^{\mathrm{p}} u}\left[\alpha \bar{B}_{i}(u)-\frac{\gamma_{i} \beta^{2}}{2}\left(1-\frac{\rho^{2} \sigma^{2}}{\sigma^{2}+\lambda \sigma_{Y}^{2}}\right) \bar{b}_{i}^{2}(u)-\frac{\left(\mu-r+\lambda \mu_{Y}\right) \rho \beta \sigma_{\bar{b}_{i}}(u)}{\sigma^{2}+\lambda \sigma_{Y}^{2}}\right. \\
& +k^{\mathbb{P}} C_{i}(u)-\left(k^{\mathbb{P}}-\frac{\vartheta}{\zeta}\right)\left(c_{i}(u)-\bar{c}_{i}(u)\right)+\frac{\left(\mu-r+\lambda \mu_{Y}\right)^{2}}{2 \gamma_{i}\left(\sigma^{2}+\lambda \sigma_{Y}^{2}\right)}+\frac{k^{\mathbb{P}}}{2 \gamma_{i}} \\
& \left.-\frac{\vartheta}{\gamma_{i} \zeta}+\frac{\vartheta^{2}}{2 \gamma_{i} \zeta^{2} k^{\mathbb{P}}}\right] \mathrm{d} u .
\end{aligned}
$$

From symmetry, it follows that

$$
\begin{aligned}
A_{i}(t)= & A_{j}(t)=\bar{A}_{i}(t)=\bar{A}_{j}(t)=a_{i}(t)=a_{j}(t)=\bar{a}_{i}(t)=\bar{a}_{j}(t), \\
B_{j}(t)= & b_{j}(t)=\bar{B}_{j}(t)=\bar{b}_{j}(t)=-\frac{1-\kappa_{j}}{r}\left(e^{r(T-t)}-1\right), \\
c_{j}(t)= & -\left(\alpha-\frac{\left(\mu-r+\lambda \mu_{Y}\right) \rho \beta \sigma}{\sigma^{2}+\lambda \sigma_{Y}^{2}}\right) \frac{1-\kappa_{j}}{r^{2}}\left(e^{r(T-t)}-1\right) \\
& -\left(\alpha-\frac{\left(\mu-r+\lambda \mu_{Y}\right) \rho \beta \sigma}{\sigma^{2}+\lambda \sigma_{Y}^{2}}\right) \frac{1-\kappa_{j}}{r}(t-T)-\frac{\left(\mu-r+\lambda \mu_{Y}\right)^{2}}{\gamma_{j}\left(\sigma^{2}+\lambda \sigma_{Y}^{2}\right)}(t-T),
\end{aligned}
$$




$$
\begin{aligned}
C_{j}(t)= & \left(\alpha-\frac{\left(\mu-r+\lambda \mu_{Y}\right) \rho \beta \sigma}{\sigma^{2}+\lambda \sigma_{Y}^{2}}\right)\left[-\frac{1-\kappa_{j}}{r^{2}} e^{r(T-t)}-\frac{1-\kappa_{j}}{r}(t-T)+\frac{1-\kappa_{j}}{r^{2}}\right] \\
& +\frac{\gamma_{j} \beta^{2}}{2}\left(1-\frac{\rho^{2} \sigma^{2}}{\sigma^{2}+\lambda \sigma_{Y}^{2}}\right)\left[-\frac{\left(1-\kappa_{j}\right)^{2}}{2 r^{3}} e^{2 r(T-t)}+\frac{2\left(1-\kappa_{j}\right)^{2}}{r^{3}} e^{r(T-t)}\right. \\
& \left.+\frac{\left(1-\kappa_{j}\right)^{2}}{r^{2}}(t-T)+\frac{\left(1-\kappa_{j}\right)^{2}}{2 r^{3}}-\frac{2\left(1-\kappa_{j}\right)^{2}}{r^{3}}\right]-\frac{\left(\mu-r+\lambda \mu_{Y}\right)^{2}}{2 \gamma_{j}\left(\sigma^{2}+\lambda \sigma_{Y}^{2}\right)}(t-T), \\
\bar{c}_{j}(t)= & e^{(\vartheta t / \zeta)} \int_{t}^{T} e^{-(9 t / \zeta)}\left[\left(\alpha-\frac{\left(\mu-r+\lambda \mu_{Y}\right) \rho \beta \sigma}{\sigma^{2}+\lambda \sigma_{Y}^{2}}\right) \bar{b}_{j}(u)+\frac{\left(\mu-r+\lambda \mu_{Y}\right)^{2}}{\gamma_{j}\left(\sigma^{2}+\lambda \sigma_{Y}^{2}\right)}+\frac{k^{\mathbb{P}}}{\gamma_{j}}-\frac{2 \vartheta}{\gamma_{j} \zeta}+\frac{\vartheta^{2}}{\gamma_{j} \zeta^{2} k^{\mathbb{P}}}\right] \mathrm{d} u, \\
\bar{C}_{j}(t)= & e^{k^{\mathbb{P}} t} \int_{t}^{T} e^{-k^{\mathbb{P}} u}\left[\alpha \bar{B}_{j}(u)-\frac{\gamma_{j} \beta^{2}}{2}\left(1-\frac{\rho^{2} \sigma^{2}}{\sigma^{2}+\lambda \sigma_{Y}^{2}}\right) \bar{b}_{j}^{2}(u)-\frac{\left(\mu-r+\lambda \mu_{Y}\right) \rho \beta \sigma_{\bar{b}_{j}}(u)}{\sigma^{2}+\lambda \sigma_{Y}^{2}}\right. \\
& +k^{\mathbb{P}} C_{j}(u)-\left(k^{\mathbb{P}}-\frac{\vartheta}{\zeta}\right)\left(c_{j}(u)-\bar{c}_{j}(u)\right)+\frac{\left(\mu-r+\lambda \mu_{Y}\right)^{2}}{2 \gamma_{j}\left(\sigma^{2}+\lambda \sigma_{Y}^{2}\right)}+\frac{k^{\mathbb{P}}}{2 \gamma_{j}} \\
& \gamma_{j} \zeta^{+} \frac{\vartheta^{2}}{\left.2 \gamma_{j} \zeta^{2} k^{\mathbb{P}}\right] \mathrm{d} u .} \\
&
\end{aligned}
$$

By equations (B.2), (B.3), (B.9), (B.17)-(B.20), (B.22), (B.23), (B.30), (B.31), and (B.39)-(B.48), we can obtain the results in Theorem 2.

\section{Data Availability}

The data used to support the findings of this study are available from the corresponding author upon request.

\section{Conflicts of Interest}

The authors declare that they have no conflicts of interest.

\section{References}

[1] C. Hipp and M. Plum, "Optimal investment for insurers," Insurance: Mathematics and Economics, vol. 27, no. 2, pp. 215-228, 2000.

[2] S. David Promislow and V. R. Young, "Minimizing the probability of ruin when claims follow brownian motion with drift," North American Actuarial Journal, vol. 9, no. 3, pp. 110-128, 2005.

[3] M. A. Milevsky, K. S. Moore, and V. R. Young, "Asset allocation and annuity-purchase strategies to minimize the probability of financial ruin," Mathematical Finance, vol. 16, no. 4, pp. 647-671, 2006.

[4] S. Chen, Z. Li, and K. Li, "Optimal investment-reinsurance policy for an insurance company with var constraint," Insurance: Mathematics and Economics, vol. 47, no. 2, pp. 144-153, 2010.

[5] E. Bayraktar and Y. Zhang, "Minimizing the probability of lifetime ruin under ambiguity aversion," SIAM Journal on Control and Optimization, vol. 53, no. 1, pp. 58-90, 2015.
[6] N. Wang, "Optimal investment for an insurer with exponential utility preference," Insurance: Mathematics and Economics, vol. 40, no. 1, pp. 77-84, 2007.

[7] L. Bo and A. Capponi, "Optimal investment in credit derivatives portfolio under contagion risk," Mathematical Finance, vol. 26, no. 4, pp. 785-834, 2016.

[8] Ł. Delong, "Optimal investment for insurance company with exponential utility and wealth-dependent risk aversion coefficient," Mathematical Methods of Operations Research, vol. 89, no. 1, pp. 73-113, 2019.

[9] W. Yuan and S. Lai, "Family optimal investment strategy for a random household expenditure under the CEV model," Journal of Computational and Applied Mathematics, vol. 354, pp. 1-14, 2019.

[10] D. Li, X. Liu, and H. Liu, "Optimal investment strategy for a family with a random household expenditure under the CEV model," Communications in Statistics - Theory and Methods, vol. 19, pp. 1-25, 2020.

[11] H. Markowitz, "Portfolio selection*," The Journal of Finance, vol. 7, no. 1, pp. 77-91, 1952.

[12] D. Li and W.-L. Ng, "Optimal dynamic portfolio selection: multiperiod mean-variance formulation," Mathematical Finance, vol. 10, no. 3, pp. 387-406, 2000.

[13] X. Y. Zhou and D. Li, "Continuous-time mean-variance portfolio selection: a stochastic LQ framework," Applied Mathematics and Optimization, vol. 42, no. 1, pp. 19-33, 2000.

[14] N. Bäuerle, "Benchmark and mean-variance problems for insurers," Mathematical Methods of Operations Research, vol. 62, no. 1, pp. 159-165, 2005.

[15] L. Bai and H. Zhang, "Dynamic mean-variance problem with constrained risk control for the insurers," Mathematical Methods of Operations Research, vol. 68, no. 1, pp. 181-205, 2008. 
[16] J. Bi and J. Guo, "Optimal mean-variance problem with constrained controls in a jump-diffusion financial market for an insurer," Journal of Optimization Theory and Applications, vol. 157, no. 1, pp. 252-275, 2013.

[17] T. Björk, M. Khapko, and A. Murgoci, "On time-inconsistent stochastic control in continuous time," Finance and Stochastics, vol. 21, no. 2, pp. 331-360, 2017.

[18] Y. Zeng and Z. Li, "Optimal time-consistent investment and reinsurance policies for mean-variance insurers," Insurance: Mathematics and Economics, vol. 49, no. 1, pp. 145-154, 2011.

[19] Z. Li, Y. Zeng, and Y. Lai, "Optimal time-consistent investment and reinsurance strategies for insurers under Heston's SV model," Insurance: Mathematics and Economics, vol. 51, no. 1, pp. 191-203, 2012.

[20] C. Zhang and Z. Liang, "Optimal time-consistent reinsurance strategies for mean-variance insurers under thinning dependence structure," Stochastic Analysis and Applications, vol. 39, no. 2, pp. 195-223, 2021.

[21] T. R. Bielecki and I. Jang, "Portfolio optimization with a defaultable security," Asia-Pacific Financial Markets, vol. 13, no. 2, pp. 113-127, 2006.

[22] D. Li, X. Rong, H. Zhao, and B. Yi, "Equilibrium investment strategy for dc pension plan with default risk and return of premiums clauses under CEV model," Insurance: Mathematics and Economics, vol. 72, pp. 6-20, 2017.

[23] H. Zhao, Y. Shen, and Y. Zeng, "Time-consistent investmentreinsurance strategy for mean-variance insurers with a defaultable security," Journal of Mathematical Analysis and Applications, vol. 437, no. 2, pp. 1036-1057, 2016.

[24] Q. Zhang and P. Chen, "Time-consistent mean-variance proportional reinsurance and investment problem in a defaultable market," Optimization, vol. 67, no. 5, pp. 683-699, 2018.

[25] G.-E. Espinosa and N. Touzi, "Optimal investment under relative performance concerns," Mathematical Finance, vol. 25, no. 2, pp. 221-257, 2015.

[26] J. Driessen, "Is default event risk priced in corporate bonds?" Review of Financial Studies, vol. 18, no. 1, pp. 165-195, 2004.

[27] D. Duffie and K. J. Singleton, Credit Risk. Pricing, Measurement, and Management, Princeton University Press, Princeton, NJ, USA, 2012.

[28] S. Kusuoka, "A remark on default risk models," Advances in Mathematical Economics, vol. 1, pp. 69-82, 1999.

[29] F. Yu, "Modeling expected return on defaultable bonds," Journal of Fixed Income, vol. 12, no. 2, pp. 69-81, 2002. 